Revue

Revue de l'histoire des religions

de Ihistoire des religions

$2 \mid 2008$

La mort et l'émotion. Attitudes antiques

\title{
Tristesse rituelle et lamentations funéraires en
} Egypte ancienne

Ritual expressions of sadness and weeping in ancient Egyptian mourning

\section{Youri Volokhine}

\section{OpenEdition}

\section{Journals}

Édition électronique

URL : http://journals.openedition.org/rhr/6043

DOI : $10.4000 /$ rhr.6043

ISSN : 2105-2573

\section{Éditeur}

Armand Colin

Édition imprimée

Date de publication : 1 avril 2008

Pagination : 163-197

ISSN : 0035-1423

Référence électronique

Youri Volokhine, "Tristesse rituelle et lamentations funéraires en Egypte ancienne », Revue de l'histoire des religions [En ligne], 2 | 2008, mis en ligne le 01 avril 2011, consulté le 10 décembre 2020. URL http://journals.openedition.org/rhr/6043 ; DOI : https://doi.org/10.4000/rhr.6043 


\section{Tristesse rituelle et lamentations funéraires en Égypte ancienne}

Dans la culture de l'Égypte ancienne, les rites de deuil sont associés à un ensemble de manifestations émotionnelles exécutées en public. Ces émotions ritualisées impliquent non seulement l'ensemble des participants aux rites funéraires, mais encore plus particulièrement le groupe des "pleureuses ». Différentes manifestations d'affliction sont à observer: postures et paroles de tristesse, ou démonstrations bruyantes (pleurs et cris) et organisées. Plusieurs exemples concernant les cérémonies privées et aussi les rites de deuil collectif sont examinés ici. Le modèle de la résolution symbolique et rituelle de l'expérience du deuil fourni par le mythe osirien, ainsi que la déploration rituelle d'Osiris, est également discuté.

\section{Ritual expressions of sadness and weeping in ancient Egyptian mourning}

In ancient Egyptian culture, mourning rites are associated with public expressions of emotions. These ritualized emotional manifestations concern the participants of the funerary rites as a whole, but even more specifically the feminine group of "weepers". Among this group, some distinctive expressions of affliction can be observed: attitudes of sadness supported by particular words, or noisy and clearly organized demonstrative weeps and screams. In the present article, a choice of examples chosen among private ceremonies are examined and put in contrast with collective mourning rites. The symbolic and ritual model proposed in this context by the Osirian myth, as well as the ritual lament of this god, shall also be taken into account. 
Au terme de sa célèbre étude sur la « représentation collective de la mort », Robert Hertz décrivait le deuil comme étant « à l'origine la participation nécessaire des survivants à l'état mortuaire de leur parent; il dure aussi longtemps que cet état lui-même ${ }^{1}$. Cette «participation nécessaire », pertinemment soulignée par le jeune sociologue, est universellement au cœur de la ritualité de la mort. Une ritualité qui consiste en une «mise en scène » (autant qu'en une « mise en sens »). Pour Patrick Baudry, la ritualité funéraire - à l'instar de toute ritualité - pose d'emblée un écart avec «soimême ». On « fait du rite », on théâtralise, c'est-à-dire que l'on adopte un comportement scénographié et prescrit. Dès lors, la question se pose de savoir si la ritualité funéraire n'est « essentiellement qu'une affaire de gestualité et de mots mis autour $»^{2}$. Une théâtralité de la douleur, donc, à laquelle toute culture donne une coloration particulière. Cette ritualité de la mort implique partout un engagement physique de la communauté des participants, les «deuillants»: cris, pleurs, démonstrations gestuelles expansives ou au contraire postures d'abattement, tristesse contenue, muette, ou exprimée ; elle implique aussi des modèles, des mythes qui fondent l'expérience de la mort, de la perte, de la disparition et de sa résolution symbolique. Une «mise en sens » que je propose ici d'aborder dans le cadre de la culture de l'Égypte ancienne.

\section{ÉMOTIONS ÉGYPTIENNES}

En Égypte ancienne, le deuil se présente d'emblée comme un comportement socialement prescrit impliquant des états émotionnels. L'accès à la constituante émotionnelle de cette expérience pose - tout comme dans d'autres cultures disparues - différentes difficultés découlant en premier lieu de la nature lacunaire de la documentation.

1. Robert Hertz, «Contribution à une étude sur la représentation collective de la mort », Année sociologique X, 1907, texte repris dans Sociologie religieuse et folklore, Paris, 1928.

2. Patrick Baudry, La place des morts. Enjeux et rites, L'Harmattan, Paris, 2006, p. 32-33. 
Nonobstant cette difficulté documentaire plusieurs études ont montré que certains points d'une «émotivité » égyptienne pouvaient être mis en lumière ${ }^{3}$. D'une façon générale, en ce qui concerne la question des émotions, il convient de s'interroger sur les possibles définitions égyptiennes d'une intériorité émotionnelle ${ }^{4}$, une approche qui passe notamment par la compréhension de la notion «d'intérieur- $j b$ » (vocable traduit aussi par «cœur»), un organe qui constituait le «fors de l'homme » 5 et qui pourrait être considéré comme le siège des émotions. D'autre part, il faut constater qu'en Égypte pharaonique l'expression des émotions est soumise à des règles spécialement contraignantes. Au sein d'une société du « regard mutuel ${ }^{6}$ (comparable à cet égard à celle de la Grèce antique analysée par Jean-Pierre Vernant), l'Égyptien se définit d'abord par rapport à sa position sociale; il en découle que l'expression des sentiments intimes ne trouve pas de place dans le genre dit « autobiographique », pourtant traditionnellement réservé à la présentation de l'individu 7 . En

3. Il n'existe pas de monographie générale sur les émotions et l'expression des sentiments en Égypte ancienne; $c f$. Brigitte Altenmüller, s.v. « Gefühlsbewegungen », Lexikon der Ägyptologie II, Harrassowitz, Wiesbaden, 1977, col. 508-510.

4. Jan Assmann, s.v. «Persönlichkeitsbegriff und -bewusstsein», Lexikon des Ägyptologie IV, 1982, col. 963-978.

5. Concept clé dont la compréhension est déterminante pour aborder le thème des émotions, que celui du « coeur- $j b$ », vocable désignant en égyptien le siège des émotions, l'homme intérieur : voir Jan Assmann, «Zur Geschichte des Herzen im Alten Ägypten», in Die Erfindung des Inneren Menschen, Studien zum Verstehen fremder Religionen 6, J. Assmann et T. Sundermeier éds., Gütersloh, 1993, p. 81-113 ; rappel de la bibliographie essentielle par H. Brunner, s.v. «Herz», Lexikon der Ägyptolgie II, 1977, col. 1158-1167; l'étude de Alexandre Piankoff, Le «cœur» dans les textes égyptiens depuis l'Ancien Empire jusqu'à la fin du Nouvel Empire, Geuthner, Paris, 1930 fournit des références utiles, mais est datée. Sur le statut physique du $j b, c f$. les lignes éclairantes de Thierry Bardinet, Les papyrus médicaux de l'Égypte pharaonique, Fayard, Paris, 1995 , p. 68-80.

6. Baudoin Van de Walle, «Le sens et la vertu du regard dans la mentalité égyptienne», Mélanges offerts à Jean Vercoutter, Paris, 1985, p. 365-374.

7. Je signale ici quelques études générales sur le vocabulaire des textes autobiographiques dans lesquelles l'on trouvera de nombreuses expressions liées aux émotions : Jozef Janssen, De Traditioneele Egyptische Autobiografie voor het Nieuwe Rijk, 2 vol., Leiden, 1946 ; Denise M. Doxey, Egyptian nonroyal epithets in the Middle Kingdom. A Social and Historical Analysis, Probleme 
revanche, les Égyptiens ont soigneusement codifié les cadres narratifs et iconographiques dans lesquels s'expriment les émotions dites « de base » (colère, peur, surprise, dégoût, joie, tristesse), tout en ne connaissant aucun terme générique pour le concept «d'émotion». Les scènes de guerre, par exemple, illustrent par le texte et l'image différentes configurations de la peur ${ }^{8}$, tandis que les textes littéraires présentent à maintes reprises des situations émotionnelles intenses ${ }^{9}$. On a pu mettre en relation des émotions telles que décrites par les textes et telles qu'attestées par l'iconographie ${ }^{10}$, ou s'intéresser, par exemple, au champ sémantique de verbes comme « rire » ou «pleurer ${ }^{11}$. Une configuration intéressante, où sources textuelles et iconographiques se croisent, se rencontre tout particulièrement dans le cadre funéraire, au sein duquel la ritualisation des émotions s'exprime de plusieurs manières. On y observe l'existence de jeux liturgiques, exprimés dans les textes et les images, et de comportements rituellement prescrits (paroles et gestes), donnant une forme

der Ägyptologie 12, Leiden, 1998 ; Jeanette A. Taylor, An Index of Male nonroyal Egyptian Titles, Epithets and Phrases of the $18^{\text {th }}$ dynasty, London, 2001 ; Karl Jansen-Winkeln Sentenzen und Maximen in den Privatinschriften der ägyptische Spätzeit, Achet. Schriften zur Ägyptologie, 1, Berlin, 1999. Cf. également Miriam Lichtheim, Maat in Ancient Egyptian Autobiographies, and related Studies, OBO 120, 1992.

8. Jan Zandee, Death as an Enemy, according to Ancient Egyptian Conceptions, Brill, Leiden, 1960. Voir la notice très riche de Jan Assmann, s.v. Furcht, Lexikon der Ägyptologie II, 1977, col. 359-367. Pas d'études systématiques sur le sujet, si ce n'est Y. Zaniolo de Vazquez-Presedo, Elemente des Schreckens im Alten Ägypten, Diss. Göttingen, 1958 (cité par Assmann).

9. Sur l'expression littéraire du sentiment amoureux : Bernard Mathieu, La poésie amoureuse de l'Égypte ancienne, Bibliothèque d'Étude de l'IFAO 115, Le Caire, 1996.

10. V. A. Donohue, «A gesture of submission», Studies in Pharaonic Religion and Society in honour of J. Gwyn Griffith, 1992, p. 82-114. Erika Feucht, «Ein Motiv der Trauer», Studien zur Sprache und Religion Ägyptens II, Fest. Westendorf, Göttingen, 1984, p. 1103-1108. Sur l'affliction, cf. aussi Serge Sauneron, Kêmi 10, 1949, p. 75-80. Sur la gestuelle, voir surtout Brigitte Dominicus, Gesten und Gebärden in Darstellungen des Alten und Mittleren Reiches, SAGA 10, 1994.

11. Waltraud Guglielmi, « Lachen und weinen in Ethik, Kult und Mythos der Ägypter », CdE LV, 1980, p. 69-86. eadem, s.v. «Lachen », Lexikon der Ägyptologie III, 1980, col. 907-908. 
et un contexte définis d'expression au chagrin. Dans ce cadre, je propose de cerner le thème des lamentations funéraires, des manifestations du chagrin ${ }^{12}$ qui, en Égypte comme partout ailleurs, s'expriment au sein des pratiques collectives du deuil ${ }^{13}$. Ce dossier, que je ne peux bien entendu que survoler au sein de la présente mise au point, s'inscrit dans le vaste domaine constitué par l'idéologie de la mort en Égypte ancienne ${ }^{14}$.

J'ai choisi de présenter pour illustrer mon propos des extraits de textes couvrant une très large chronologie et de confronter de cette façon, en l'espace de quelques pages, des idées traversant près de trois millénaires ; cette méthode, souvent risquée dans d'autres contextes culturels, s'avère néanmoins adaptée en ce qui concerne la documentation qui nous est parvenue de l'Égypte pharaonique ${ }^{15}$.

\section{DES PRATIQUES CONTRAIGNANTES :}

\section{LES MANIFESTATIONS DE DEUIL AUX FUNÉRAILLES DE L'APIS}

Au sein des rites de la mort en Égypte, une place est dévolue aux lamentations et aux pleurs. Dans le cortège funéraire (tel que l'atteste l'iconographie), la monstration de l'affliction est souvent spécifiquement assumée par un cortège de «professionnels » (les «pleureuses », personnages dont nous discuterons ci-après). Mais avant de traiter des funérailles privées, penchons-nous sur des

12. Cf. Erich Lüddeckens, Untersuchungen über religiösen Gehalt, Sprache und Form der ägyptischen Totenklagen, MDAIAK 11, 1943.

13. Wolfhart Westendorf, sv. «Trauer », Lexikon der Ägyptologie VI, 1986, col. 744-745.

14. Pour une approche générale de la mort dans la culture égyptienne ancienne, on lira en dernier lieu la somme de Jan Assmann, Mort et au-delà dans l'Égypte ancienne, Éditions du Rocher, Paris, 2003. Du même auteur, Images et rites de la mort, Cybèle, Paris, 2000.

15. En effet, les grandes lignes des structures mythologiques (voire rituelles) mises en place en tout cas dès la moitié du troisième millénaire avant notre ère perdurent durant toute l'histoire pharaonique ; cette situation rend possible ce regard enjambant les siècles, un regard rétrograde que les Égyptiens pratiquaient d'ailleurs eux-mêmes, puisqu'ils s'alimentaient périodiquement aux sources de leur tradition, en allant puiser et piocher dans des textes anciens. 
circonstances de deuil différentes. Le premier cas que je souhaiterais présenter ici concerne non pas le « deuil privé » (c'est-à-dire familial) mais « le deuil collectif » ${ }^{16}$. Il se trouve qu'un dossier archéologique cohérent révèle des attitudes collectives de lamentations, associées aux funérailles du taureau Apis à Memphis. L'importance des cérémonies accompagnant ces funérailles n'avait, du reste, aucunement échappé aux auteurs classiques. Ainsi, chez Diodore de Sicile :

«Si une bête meurt, ils mènent le deuil comme s'ils avaient perdu des enfants chéris et l'ensevelissent non pas selon leurs moyens mais bien au-delà de la valeur de leur fortune. Ainsi, après la mort d'Alexandre, quand Ptolémée fils de Lagos venait de prendre en mains l'Égypte, il se trouva qu'à Memphis l'Apis mourut de vieillesse ; celui qui en avait la charge dépensa, pour l'ensemble des funérailles, les ressources dont il disposait, qui étaient très importantes, et emprunta en outre à Ptolémée cinquante talents d'or. ${ }^{17}$ »

«(...) Une fois qu'on l'a trouvé (= le veau qui succédera au taureau défunt), les masses mettent un terme au deuil (...). ${ }^{18}$ »

Diodore a été frappé du fait que les Égyptiens semblaient vivre le deuil de l'animal sacré comme s'il s'agissait de celui d'un être cher. L'analogie entre le deuil éprouvé pour un proche et celui d'un animal, fût-il sacré, avait peut-être de quoi surprendre le lecteur grec, et a dû contribuer à stigmatiser la zoolâtrie suspecte des Égyptiens. Quoi qu'il en soit, la documentation égyptienne révèle en l'occurrence des pratiques personnelles assez contraignantes (privation, jeûne, lamentations), mais rituellement exécutées. À l'occasion des funérailles du taureau Apis, le deuil est collectif : une foule de « deuillants » accompagne le cadavre du taureau sacré, le conduisant vers la nécropole (les catacombes des taureaux du Sérapeum) qui domine la plaine memphite ; divers indices montrent que lors de cette cérémonie une certaine suppression des classes sociales pouvait être revendiquée (en tant que signe de deuil). En outre, on constate dans cette documentation un recours fréquent à un vocabulaire

16. En l'occurrence, une distinction catégorielle entre ces deux circonstances d'expression de la tristesse n'implique pas une différence radicale des attitudes, l'une et l'autre procédant, comme nous le verrons, d'un modèle commun de gestion de la mort (l'expérience de la mort d'Osiris).

17. Diodore I. LXXIV, trad. M. Casevitz.

18. Diodore I. LXXXV, trad. M. Casevitz. 
« émotionnel»: des pratiques paroxystiques et des manifestations «théâtrales » de chagrin sont précisément décrites. Au cours de ce chagrin public, les éléments du deuil et de sa dramatisation sont soigneusement codifiés et ritualisés : il s'agit d'une mise en scène de la tristesse «sur la place publique »- ou plus exactement dans l'espace de la nécropole, et durant le temps prescrit pour le deuil. Nous pouvons nous en rendre compte en lisant quelques extraits choisis au sein de la riche documentation des stèles du Sérapeum :

«Le "bienheureux" (jmakh) auprès d'Apis-Osiris, qui préside aux Occidentaux, le fils du roi Khnoum-jb-Rê (= Amasis), qu'il vive éternellement, Psammétique (...) il dit : "Je suis un serviteur véritable et favori du grand dieu (Apis). J'ai 'orné mon corps' ${ }^{19}$ lors de la 'montée au ciel' (d'Apis) ; je me suis privé de pain et d'eau jusqu'à l'accomplissement des quatre jours. J'étais nu et je tremblais ${ }^{20}$ (assis) sur mon derrière ; j'ai été parmi les 'affligés' (ou 'les pauvres' ?) ${ }^{21}$ étant 'en deuil' 22 et m'affligeant ${ }^{23}$. Aucune nourriture (litt. 'chose') ne descendit dans mon ventre à l'exception de pain, d'eau et de légumes jusqu'à l'accomplissement des soixante-dix jours, lorsque le grand dieu sortit de la Ouabet et qu'il occupa sa grande tombe dans la nécropole, dans le désert occidental de Hout-ka-Ptah (= Memphis) : alors, je fus devant lui en me lamentant ${ }^{24}[\ldots]$ en l'an [...] qu'il vive à jamais, fermement établi [...] de Sa Majesté" » (Stèle Louvre SIM. 403425).

«Le père-divin de Ptah, le "connu-du-roi" de Sokaris, le gardien du terrain de l'Apis vivant, son serviteur véritable, son favori, qui fait ce qu'il (= Apis) désire dans toutes ses fêtes. Celui qui a suivi la Majesté (du dieu) en se lamentant et en "ornant son corps" lorsqu'il (= Apis) monta au ciel (= lorsqu'il mourut). Celui qui s'est couché par terre, se privant de pain et d'eau, cessant de ceindre ses reins d'un pagne jusqu'à

19. Shet haou, « orner (son) corps », porter un vêtement funéraire, "prendre le deuil »; cf. texte C, Vercoutter, op. cit., note 25, p. 29.

20. sed cf. prob. sedâ « trembler», Wb IV, 365-366.

21. shouâou « les pauvres »Wb IV, 435.3-7.

22. shet, désigne un état ou une action indéterminé, peut-être lié au port d'un vêtement spécifique, en rapport au deuil, $c f$. shet haou, ci-dessus.

23. sâmet, $W b$ I V, 18.10 : action indéterminée accomplie lors des funérailles ; le terme sert aussi à désigner de façon générale le deuil.

24. $j h$.

25. Provenance : Sérapeum de Memphis (fouilles de Mariette). Datation : $\mathrm{XXVI}^{\mathrm{e}}$ dynastie, règne d'Amasis ( 570-525 av. J.-C .) ; voir Jean Vercoutter, Textes biographiques du Sérapeum de Memphis. Contribution à l'étude des stèles votives du Sérapéum, Bibliothèque de l'EPHE IVe section 316, Paris, 1962, p. 37-43 (texte E). 
ce que vînt le jour de "se réunir à la terre" (= l'enterrement), où la Majesté (du dieu) reposa dans le Kem (= le Sérapeum) en vie, santé force. Le prophète d'Isis et le prophète de Nephthys, le chancelier Ouah-jb-Rêounnefer, fils de Horsaisis, né de la dame Nestanefert » (Stèle Louvre SIM. $4100^{26}$ ).

"Le "bienheureux" auprès d'Apis-Osiris, le prêtre-houn de Bastet, Panehesy, dont le beau nom est Neferibrê-Seneb, fils de Pathenef, qui a été mis au monde par Iahneferou. Il dit : "Je suis un serviteur véritable, le favori du grand dieu. J'ai 'orné mon corps' lors de sa montée au ciel. Je me suis privé de pain et d'eau. J'ai passé les soixante-dix jours en étant en deuil et en m'affligeant, une bandelette-pyr passée autour du $\mathrm{cou}^{27}$, et je poussais des plaintes (ihy) chaque jour. Puis, j'ai fait que ce dieu (= Apis) sorte de la Ouabet et qu'il occupe sa tombe (litt. : sa place) dans le bel occident du Kemet (= Sérapeum)" »(Stèle Louvre SIM. $4051^{28}$ ).

«Le "bienheureux" auprès d'Apis-Osiris qui préside aux Occidentaux, l'administrateur des biens du temple d'Imet (Nebesheh) (...) Neferibrê (...), il dit : "Je suis un serviteur véritable, le favori du grand dieu. J'ai 'orné mon corps' lors de sa montée au ciel. Je me suis privé de pain et d'eau jusqu'à l'accomplissement des quatre jours. Je m'habillai comme un pauvre ${ }^{29}$ et je fus parmi les hommes de peu ${ }^{30}$ à prendre le deuil ${ }^{31}$, à m'affliger et à crier. Aucune nourriture n'est descendue dans mon ventre, si ce n'est de l'eau et des légumes, jusqu'à l'accomplissement des soixante-dix jours, jusqu'à ce que le grand dieu quitte la Ouabet. Puis, Sa Majesté (= Apis) sortit vers la tente-depurification $^{32}$; lorsqu'elle descendit dans sa barque, j'étais à l'avant ${ }^{33}$, en train de gémir avec les 'pauvres'. On ne distinguait pas mon rang du leur, en regard de l'offrande-abet et de l'offrande-oudemet. J'ai fait ces choses car j'étais instruit de ta puissance. La conduite de ma vie était dans ta main ; une sépulture auprès de toi sera ma récompense de par toi, après une longue vie dans les faveurs du Maître du pays. Tu es la

26. Provenance : Sérapeum de Memphis. Datation : règne d'Amasis (vraisemblablement an 5). Voir Vercoutter, op. cit., p. 27-33 (texte C).

27. Voir Philippe Collombert, «La bandelette-pyr au cou des deuillants », $R d E 235$ (2006), p. 235-237, qui corrige la traduction de Vercoutter (op.cit.), p. 44-46, « jusqu'à ce que ma gorge fut voilée ».

28. Provenance : Sérapeum de Memphis. Datation : règne d'Amasis. Voir Vercoutter, op. cit., p. 44-47 (texte F).

29. $n f s$, litt. " petit», homme du peuple, mais aussi « humble».

30. hourou, homme du peuple, $W b$ III, 55.8.

31. her snm herng.

32. Un des édicules liés aux rites de momification.

33. Litt. « sous la proue » (de la barque divine), c'est-à-dire en tête de la procession. 
vie, ô Maître de l'éternité ; c'est la pérennité que d'être auprès de toi" » (Stèle Louvre SIM. 411034).

Ces documents révèlent l'existence d'un rite de deuil particulier dans l'Égypte tardive, au cours duquel différentes expériences personnelles recouvrent un modèle commun. Ce rite d'affliction, accompli tout au long de la durée conventionnelle des rites de momification de l'Apis (soixante-dix jours), comprend des privations de nourriture, ainsi que le port de vêtements spécifiques : bandelette de deuil, vêtements dits « de pauvre ». Le port d'un vêtement spécifique, qui de plus est associé à une tenue dépourvue de tout signe ostentatoire de richesse ou de luxe, est aussi nettement revendiqué par les « deuillants » (parmi lesquels on rencontre même le prince héritier). Toutes ces pratiques concourent à infliger au corps une contrainte, à lui imposer un type de comportement que l'on rencontre très fréquemment dans les rites de deuil (voir ci-dessous). La participation aux funérailles de l'Apis et l'adoption à cette occasion d'une conduite rituellement prescrite sont de nature à favoriser le participant ; sa piété ainsi démontrée est agréable au dieu, et il est assuré d'en retirer un grand bénéfice. L'adoption d'un comportement de tristesse démonstrative prend ainsi place dans l'ensemble des rites concernant la mort du taureau Apis (et non seulement ses funérailles proprement dites). Certains «deuillants » ont participé ${ }^{35}$ à la totalité de ces rites qui comportent - comme il sied pour tout dieu « qui meurt » ${ }^{36}$ - des lamentations prononcées dans un cadre

34. Provenance : Sérapeum. Datation : an 23 d'Amasis. Voir Vercoutter, op. cit., p. 48-58 (texte G).

35. Philippe Collombert, RdE 57, 2006, p. 236.

36. Sur ce thème frazérien, voir notamment Paolo Xella (éd.), Quando un dio muore. Morti et assenze divine nelle antiche tradizioni mediterranee, Essedue, Verona, 2001 ; Tryggve N.D. Mettinger, The Riddle of Resurrection: Dying and Rising Gods in the Ancient Near East, Coniectanea Biblica, Old Testament Series 50, Almqvist \& Wiksell, Stockholm, 2001. Cf. l'approche critique quant au concept même de « dieu qui meurt » de Jonathan Z. Smith, « Dying and Rising Gods », dans : Encyclopedia of Religion, vol. 4, New York, 1987, p. 521-527 (repris dans Drudgery Divine : On the Comparison of Early Christianities and the Religions of Late Antiquity, Chicago, 1990, chap. 4), ainsi que de Mark S. Smith, « The Death of Dying and Rising Gods in the Biblical World », Scandinavian Journal of the Old Testament 12, 1998, p. 257-313. 
liturgique ${ }^{37}$. Ces lamentations s'inscrivent, faut-il le souligner, dans le vaste dossier concernant les rites de type funèbre associés aux célébrations de la mort d'un personnage divin, dont les ramifications mènent du Tammouz oriental jusqu'à Adonis : je ne m'aventurerai toutefois pas plus avant dans ce thème comparatiste, autrefois frazérien, du « dieu qui meurt », ni sur les différentes descriptions de chagrins collectifs associés à cette occasion et que les textes antiques nous ont transmis autour d'Adonis ${ }^{38}$ ou d'Attis ${ }^{39}$.

\section{LE DEUIL DU ROI}

Revenons, un court instant, à Diodore de Sicile :

«Ce qui se passait après la mort des rois chez les Égyptiens fournissait une démonstration qui n'était point mince de la dévotion de la masse envers les chefs (...). Donc, quand un roi décédait, tous les habitants de l'Égypte menaient un deuil collectif, ils laceraient leurs vêtements, fermaient les sanctuaires, suspendaient les sacrifices et ne célébraient pas les fêtes pendant soixante-douze jours : la tête enduite de boue, une étoffe de mousseline nouée au-dessous de la poitrine, hommes et femmes indistinctement, ils parcouraient les rues par groupes de deux ou trois cents; chantant le thrène en cadence, ils décernaient les honneurs de glorification deux fois par jour, rappelant la vertu du mort ;

37. R. L. Vos, The Apis Embalming Ritual P. Vindob. 3873 (OLA 50), 1993, p. 43, 72-73.

38. Pour les lamentations lors des Adonies, $c f$. notamment Marcel Detienne, dans Dictionnaire des Mythologies, I, Yves Bonnefoy éd., Flammarion, Paris, 1981, p. 1-4. Voir le texte de Lucien (La Déesse Syrienne, VI), sur les manifestations du deuil d'Adonis à Byblos, dont certains traits sont précisément comparés au deuil de l'Apis égyptien ; et aussi la description du chagrin des femmes lors des fêtes alexandrines chez Théocrite (XV, 132-135). Sur les lamentations rituelles (pour les données factuelles), cf. James G. Frazer, Le Rameau d'Or, vol. 2, Robert Laffont, Paris, 1983, spécialement p. 214-215 et 330. Enfin, sur les pleurs signalant la mort du dieu, on se souviendra du célèbre épisode de l'annonce de la mort du "Grand Pan », accueillie par un concert de larmes, chez Plutarque (Sur la disparition des oracles, 17) : voir en dernier lieu Philippe Borgeaud, dans Exercices de Mythologies, Labor et Fides, Genève, 2004, p. $115-155$.

39. Cf. Philippe Borgeaud, La Mère des dieux. De Cybèle à la Vierge Marie, Seuil, Paris, 1996, (spécialement p. 133). 
ils n'absorbaient pas de nourriture à base d'animaux ou de froment et s'abstenaient de vin et de toute marque de luxe. ${ }^{40}$ »

Parallèlement au deuil collectif manifesté aux funérailles de l'Apis, Diodore constate là encore des cérémonies d'affliction collective, dans ce cas en faveur du pharaon défunt. Relativement peu de renseignements nous sont connus en ce qui concerne le deuil qui devait vraisemblablement frapper le pays à ce moment-là. Tout semble indiquer que la phase de transmission effective du pouvoir (durant le temps séparant le décès du roi de son ensevelissement), au moment même du deuil, devait être une phase «de tous les dangers ». Un célèbre texte littéraire du Moyen Empire, ledit « conte de Sinouhé », évoque un deuil frappant la cour royale :

«L'an XXX, le troisième mois de l'inondation, le 7, le dieu entra dans son horizon, le roi de Haute et Basse Égypte Séhétepibrê (= Amenemhat I) ; il fut enlevé au ciel et ainsi se trouva uni avec le disque solaire, et le corps du dieu s'absorba en Celui qui l'avait créé. La cour était dans le silence, les cœurs dans la tristesse ; la double porte restait close ; les courtisans étaient (accroupis) la tête sur les genoux et le peuple se lamentait. ${ }^{41} »$

Un modèle d'affliction particulier se profile ; une tristesse moins expansive que celle des pleureuses échevelées : l'abattement muet des notables, répondant aux pleurs du peuple. Cet extrait révèle en outre l'une des postures-types du deuil masculin. En effet, l'homme abattu, en proie à la tristesse ou au deuil, adopte une attitude prostrée, consacrée par l'expression « avoir la tête sur les genoux » (tep her maset ${ }^{42}$. Une telle attitude corporelle est aussi adoptée par les dieux. En effet, dans un passage du Calendrier des jours fastes et néfastes

40. I, LXXII, trad. M. Casevitz.

41. Provenance : p. Berlin 10499 et parallèles. Datation : XIII ${ }^{\mathrm{e}}$ dynastie (p. Berlin 10499), rédaction : XII ${ }^{e}$ dynastie. Voir Gustave Lefebvre, Romans et contes égyptiens, p. 5. Roland Koch, Die Erzählung des Sinuhe, Bibliotheca Aegyptiaca XVII, Bruxelles, 1990, p. 4-6 R 5-11).

42. $W b$ V, p. 265.1. Voir sur cette expression: Serge Sauneron, Kémi 10, 1969, p. 75-80 ; Etienne Drioton, BSFE 12, 1953, p. 15; Erika Feucht, dans: Studien zur Sprache und Religion Ägyptens (Fest. Westendorf), 2, 1984, p. 11041108. Giuseppe Bottini, «Pose la sua faccia tra le ginocchia, 1 Re 18,42 et paralleli estrabiblici », Liber Annus, Studium Biblicum Franciscanum 32, Jerusalem, 1982, p. 73-84 ; Elke Blumenthal, Untersuchungen zum Ägyptische Königtum, 1970, p. 334-335 G 5.18. 
intervient une description de l'affliction divine : "Alors ces dieux (s'assirent) le visage (ou "la tête" ?) sur les genoux, (leurs) visages (tournés) en bas ${ }^{43}$. On rapprochera ce texte d'une formule liturgique consacrée à Osiris, datant du début de l'époque ptolémaïque : «Les dieux (ont) le visage sur les genoux (car) tu ne viens pas ${ }^{44}$. On retrouve ce thème de la prostration, avec des accents similaires, dans la liturgie osirienne où l'expression «nos têtes sont recourbées (ân) sur nous-mêmes », exprime également un état d'affliction ${ }^{45}$. Dans l'iconographie, la posture de l'homme dont la tête repose sur les genoux signale, elle aussi, une gestion corporelle du deuil, qui tranche parfois avec d'autres gestes d'affliction plus spectaculaires ${ }^{46}$.

Expressions quasi proverbiales, gestes figés dans l'iconographie : nous sommes loin, assurément, de l'évocation de manifestations émotionnelles spontanées. D'ailleurs, ce n'est que fort rarement que les textes semblent insister sur des sentiments intérieurs ressentis à la mort du roi par l'un de ses proches ${ }^{47}$. Toutefois, c'est une telle

43. Christian Leitz, Tagewählerei, ÄgAbh 55, 1994, p. 232, et pl. 70 p. Sallier recto XVI.5. Le parallèle du papyrus du Caire présente la variante suivante «la tête (sur) les genoux, le visage (en) bas »; Chr. Leitz, op. cit, p. 232 ; Abd el-Mohsen Bakir, The Cairo Calendar, 1966, pl. XXIII.9, p. 33.

44. A. Dembska, Studia Aegyptiaca 14, 1992, p. 114 ; (papyrus Schmitt: p. Berlin 3057, 24.9); Dembska suggère également de comprendre ici « la face sur les genoux », plutôt que « la tête sur les genoux ».

45. R. Faulkner, Bremner-Rhind, p. 3.10 (2.1) ; Pascal Vernus, Chants d'amour de l'Égypte antique, 1992, p. 102. L'expression «(avoir) la tête sur les genoux » est bien attestée dans les rites de déplorations d'Osiris, par exemple : Jean-Claude Goyon, BIFAO 65, 1967, p. 97, et p. 143 (p. Louvre I 3079, col. 110. 21).

46. Erika Feucht, op. cit., pl. 1-3. Cf. les attitudes de fatigue (tête sur les genoux), par exemple Geoffrey T. Martin, The Tomb of Horemheb, EES 55, 1989, pl. 95 [69], et pl. 125 [88].

47. Une exception notoire existe cependant : le désespoir face à la mort est figuré dans les représentations remarquables provenant de la nécropole royale de Tell el Amarna. Le pharaon Akhénaton, accompagné de son épouse, est montré en lamentation devant le lit funèbre sur lequel une jeune princesse est étendue, tandis que la cour est en deuil. Cette représentation rompt avec la norme : en effet, aucune autre représentation d'un roi en lamentation funèbre n'est connue ; de plus, la déploration se déroule ici non pas devant un sarcophage, ou durant la procession funéraire, mais directement devant le cadavre, ce corps que l'image égyptienne répugne d'ordinaire à montrer. Ces innovations stylistiques et thématiques s'inscrivent dans la nouvelle idéologie de la 


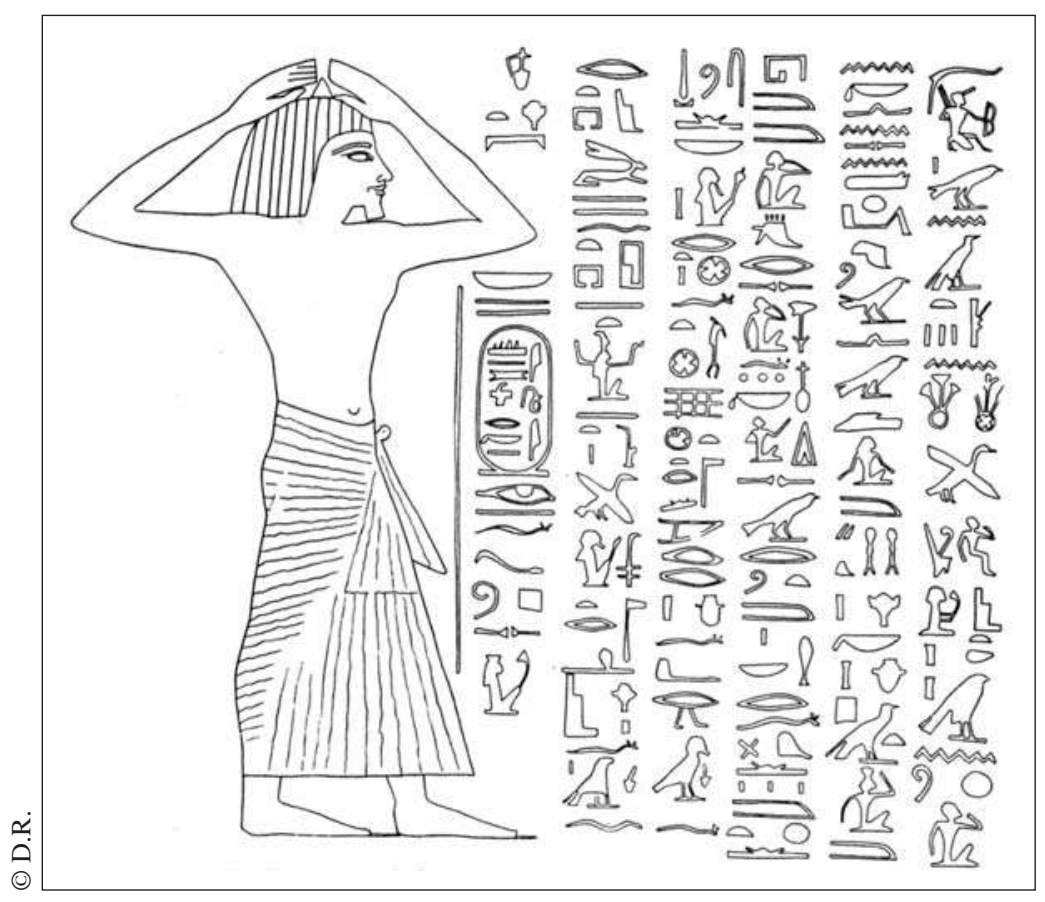

Fig. 1. Le général Pachereniset en lamentation.

D'après Victor Loret, Kêmi 9 (1942), p. 97-106.

expérience que paraît nous confier Pachereniset, un notable qui fit graver à Tanis, tout juste à l'entrée du tombeau de son roi, une inscription commémorant sa tristesse (fig. 1).

«Le général en chef des troupes de Haute et Basse Égypte, Pachereniset, fils de Hor: "Je veux me lamenter pour toi sans cesse, sans me lasser de chercher ton visage, mon esprit (jb.j) chargé de murmures $(\mathrm{hmhm})$ à évoquer ta perfection. Je veux faire qu'on trouve plus à s'enrichir dans la personnalité (hem) de mon maître que dans les avantages matériels. J'ai escorté mon maître jusqu'à sa ville, Thèbes le district divin qu'aimait son $\operatorname{cœur}^{48}$. Je veux faire que son $b a$ s'en aille à la place où il demeurera, le

mort amarnienne qui tranche passablement avec l'au-delà osirien traditionnel. Voir Geoffrey T. Martin, The Royal Tomb at El-Amarna, II, ASEM 39, 1989, pl. 58-61 ; cf. Marc Gabolde, D’Akhénaton à Toutânkhamon, Paris, 1998, pl. IV, et p. 19-21 (sur l'idéologie amarnienne de la mort).

48. La mention de la ville de Thèbes désigne ici Tanis (la «Thèbes du nord »), et non Louxor. 
Domaine-des-millions-d'année (= la tombe royale). Le roi est divinisé, reposant dans la place, tandis que son $b a$ rejoint le ciel ; le maître des deux pays, Osorkon-aimé-d'Amon, c'est pour lui qu'a agi Kapous (= la reine-mère)." 49 »

Représenté en pied devant l'inscription, le général Pachereniset accompagne les mots de cette déploration par un geste d'affliction : il porte, en signe de désarroi, les deux mains à sa tête. Les accents poignants de ce texte nous amènent à présent à envisager le référent mythologique de ce discours de deuil. En effet, l'expérience de la mort est d'abord, pour le survivant, celle de la séparation, induisant la quête impossible de l'être aimé : voilà toute la trame du mythe osirien.

\section{LA DÉPLORATION D'OSIRIS : MODÈLE DE LA PLAINTE FUNÈBRE}

La mort d'Osiris marque mythologiquement l'irruption de la mort dans le monde. Il est impossible de dissocier le mythe d'Osiris de l'image - comprenons : de l'idéologie - de la mort en Égypte ${ }^{50}$. Ce mythe met en scène une victime (Osiris) et son meurtrier (Seth). Osiris, le roi, est assassiné par son frère Seth qui convoite le pouvoir. Isis, l'épouse éplorée aura comme tâche de retrouver (et reconstituer) le corps de son époux défunt, et de concevoir avec lui un héritier légitime (Horus), qui régnera sur le trône d'Égypte tandis qu'Osiris, quoique « ressuscité », demeurera éternellement dans la Douat (= l'audelà). Comme le propose Jan Assmann, le mythe met en scène les trois points de vue principaux des Égyptiens sur la mort : la mort en tant que déchirement ; la mort en tant qu'isolement social ; et enfin la mort en tant qu'ennemi. Trois étapes narratives se dégagent : la recomposition du corps (l'intégrité physique garantie par les soins funéraires que prodiguent Isis et Nephthys); la reconstitution de la connectivité sociale (Horus, le fils, assure le culte funéraire et accède légitimement, par héritage, à la fonction royale); l'affrontement

49. Texte gravé à l'entrée du tombeau d'Osorkon II à Tanis, XXII' dynastie ( 874-850 av. J.-C.). Voir Pascal Vernus, dans : Tanis. L'or des pharaons, Paris, 1987, p. 109 ; cf. Victor Loret, Kêmi 9 (1942), p. 97-106.

50. Jan Assmann, Mort et au-delà en Égypte ancienne, Paris, 2003. 
judiciaire (Seth est débouté de sa prétention au pouvoir à l'issue d'un procès qui sanctionne sa culpabilité) et la moralisation finale (la mort est un crime, une injustice).

Le mythe osirien présente donc une vision de la mort qui l'assimile à un meurtre. C'est ainsi que la mort est assumée rituellement et devient dès lors viable (comme le propose Jan Assmann). L'expérience de la mort est sciemment vécue dans toute sa dimension tragique. Dans ce cycle, Seth représente le meurtrier : c'est dire qu'il joue le rôle de la mort, alors qu'Osiris joue celui du mort. Seth est vaincu, condamné, et proscrit. Le mythe égyptien, comme le précise Assmann, «fait ressortir non seulement le caractère brutal de la mort, mais aussi son caractère inique ${ }^{51}$. Dans ce cadre, toute mort devient une atteinte au droit (la Maât). On peut dès lors la juger (ce qui dénote, selon Assmann, une moralisation de l'idée de mort). C'est donc dans le cadre de cette moralisation et gestion particulière de la mort que s'exprime la tristesse du deuil. Le motif de la tristesse intervient à plusieurs reprises dans le mythe osirien. En premier lieu, le mythe assimile la mort du dieu à une catastrophe cosmique. Un exemple clair est offert par le papyrus Salt 825, un texte rituel de l'époque ptolémaïque consacré à la résurrection d'Osiris ; le rituel implique plusieurs prêtres spécialisés, et mentionne des rites d'envoûtement et des rites funéraires célébrés manifestement autour d'une statue d'Osiris, ainsi que des rites de protection du roi et du palais. Le texte relate qu'un grand bouleversement frappa l'Égypte consécutivement à la mort d'Osiris ${ }^{52}$ :

«(I.1) [...] ce [n'est pas] éclairé pendant la nuit, et le jour n'existe pas. Lamentation, lamentation (ihy) [dans le ciel] et la terre. Les dieux et les déesses se mettent les mains sur la tête, la terre est dévastée, le soleil ne sort pas, la lune tarde, elle n'existe pas. Le Noun vacille, la terre chavire, le fleuve n'est plus navigable. [...] entendre. Tout le monde gémit $(j k b)$ et pleure $(\mathrm{rmj})$. Les baou, les dieux, les déesses, les hommes, les akhou,

51. Assmann, op. cit., p. 117.

52. Les premières pages du manuscrit (où figure l'épisode de la mort d'Osiris) étaient encore inconnues au moment de l'édition princeps du texte par Philippe Derchain; elles ont depuis lors été retrouvées : $c f$. François René Herbin, «Les premières pages du Papyrus Salt 825 », BIFAO 88, 1988, p. 95112. 
les morts, les bestiaux petits et grands, les [...] pleurent, pleurent beaucoup $\left[\ldots . .{ }^{53}{ }^{\prime \prime}\right.$

Comme on le voit, la mort d'Osiris implique un grave déséquilibre. Elle met en péril le cosmos, constitue une rupture dans la marche de l'univers. C'est un problème qu'il faut absolument résoudre. Cette mort plonge le monde dans la tristesse, dans un chagrin qui frappe non seulement les dieux et les hommes, mais également les animaux. Le rituel va pleinement assumer cette tristesse ${ }^{54}$. En effet, les chants liturgiques de déploration du dieu défunt (prototype de tout mort), exploitent largement les motifs de la perte, du déchirement après la séparation brutale, tout autant que celui du désir de « voir » à nouveau le défunt, au-delà de cette séparation. Dès les Textes des Pyramides, la déploration du dieu mort est une tâche assumée par son épouse (et sœur) Isis, accompagnée de sa sœur Nephthys ${ }^{55}$.

« Paroles-à-dire par Isis et Nephthys. L'oiseau-hât vient, l'oiseau-djéret vient, Isis et Nephthys ; elles viennent pour étreindre leur frère Osiris, pour étreindre (ou : chercher) le roi. Elles se hâtent mutuellement. Pleure pour ton frère, Isis ! Pleure pour ton frère, Nephthys ! Isis s'est assise les mains sur la tête. Nephthys a saisi les pointes de ses seins pour son frère le roi, qui se tient (couché) sur son ventre (...). ${ }^{56}$ »

La déploration funèbre est ici manifestement assimilée aux chants lugubres de deux oiseaux : l'oiseau-hat (voir le verbe hai « se lamenter » qui procède de la même racine ${ }^{57}$ ), et l'oiseau-djéret (d'où le terme djéret « plaignante » ${ }^{58}$; les « deux-djéret » seront la

53. Philippe Derchain, Le papyrus Salt 825 (B.M. 10051), rituel pour la conservation de la vie en Égypte, Académie Royale de Belgique, Bruxelles, 1965.

54. Voir Jan Assmann, Mort et au-delà en Égypte ancienne, p. 214-224.

55. Sur le rôle conjoint des deux déesses pleureuses, $c f$. Claas J. Bleeker, «Isis and Nephthys as wailing Women », in The Sacred Bridge, Studies in the History of Religions, 7, Leiden, 1963, p. 190-205.

56. Textes des Pyramides, chapitre 535, § 1280-1281. Provenance : pyramide de Pépi I ${ }^{\mathrm{er}}$, Saqqara. Datation : VI ${ }^{\mathrm{e}}$ dynastie (circa 2330 av. J.-C.). Bibliographie : Kurth Sethe, Die Altaegyptischen Pyramidentexte, II, Leipzig, 1910, p. 219-220 ; Raymond O. Faulkner, The Ancient Egyptian Pyramids Texts, 1969, p. 203.

57. Cf. Alan H. Gardiner, in JEA 41, 1955, p. 10.

58. $W b \mathrm{~V}, 596.2-8$ (faucon), et 596.9-597.3 pour les désignations des deux pleureuses. 
désignation usuelle d'Isis et Nephthys dans leur rôle de pleureuses). C'est donc à l'épouse et à la sœur du dieu défunt que l'expression vocale de la lamentation est confiée. On soulignera ici le jeu de Nephthys. Le geste particulier qu'elle exécute - consistant à présenter ses seins au défunt - demande à être compris comme une incitation à la résurrection. En dévoilant sa poitrine, tout comme la femme qui allaite, la déesse fait du défunt un implicite nouveau-né, promis à la vie qui découle du lait nourricier. Il appert, effectivement, que ce cérémonial de lamentations comporte un aspect vivifiant. Comme l'écrit Jan Assmann, Isis et Nephthys, les «oisellespleureuses », «peuvent pleurer de façon à réveiller un mort ${ }^{59}$. Les plaintes rituelles d'Isis et de Nephthys vont faire l'objet d'une élaboration littéraire remarquable, et plusieurs exemples de livrets consignant leurs lamentations nous sont parvenus, conservés par des documents d'époque tardive ${ }^{60}$. Ces textes étaient prononcés originellement lors de liturgies sacerdotales d'accompagnement d'Osiris ${ }^{61}$, et ils ont aussi été choisis par des particuliers pour figurer dans leur trousseau funéraire. Il s'agit de textes très poétiques, dont on peut souligner la proximité stylistique et thématique avec les «chants d'amour » ${ }^{62}$. En effet, le chant de la veuve thématise la douleur de la séparation et de la perte, et son corollaire immédiat qui est le désir prégnant de (re)voir l'absent, exaltant par là même le sentiment amoureux, comme l'on va pouvoir s'en rendre compte en lisant deux extraits significatifs tirés de ce corpus :

« Les Lamentations d'Isis et de Nephthys »

«(1.1) Récitation des liturgies qu'ont faites les deux sœurs dans la demeure d'Osiris-qui-préside-à-l'Occident, le dieu grand, maître d'Abydos, le quatrième mois de la saison de l'inondation, le vingtcinquième jour (...). »

59. Assmann, op. cit., p. 218.

60. Leur élaboration remonte sans doute au Nouvel Empire.

61. Voir Jean-Claude Goyon, Le papyrus d'Imouthès fils de Psintaês au Metropolitan Museum of Arts de New York (Papyrus MMA 35.9.21), New York, 1999, spécialement p. 35-43 et p. 85-94.

62. Pascal Vernus, Chants d'amour de l'Égypte antique, Paris, 1992, publie d'ailleurs dans le même recueil poésie amoureuse et chants funèbres. Pour la poésie amoureuse, voir Bernard Mathieu, La poésie amoureuse de l'Égypte ancienne, Bibliothèque d'Étude de L'IFAO 115, Le Caire, 1996. 
«Paroles prononcées par Isis ; elle dit : (2.1) Viens vers ta demeure, viens vers ta demeure ! l'héliopolitain ; viens vers ta demeure ! tu n'as pas d'ennemi. Ô Ihy parfait, viens vers ta demeure, que je te contemple. Je suis ta sœur, que tu aimes; tu ne dois pas te séparer de moi. Ô jeune homme parfait, viens à ta demeure ! Longtemps, longtemps que je ne t'ai pas contemplé ! Mon cœur s'inquiète de toi ; mes deux yeux te cherchent; je suis en quête de toi pour te contempler. Puissé-je te contempler, puissé-je te contempler ! (...) (2.9) je suis ta sœur de par ta mère, tu ne dois pas t'éloigner de moi. Dieux et hommes, leurs visages sont (tournés) vers toi, te pleurant tous ensemble! Alors que je ne peux te voir, je t'appelle en pleurant jusqu' au haut du ciel, sans que tu entendes ma voix, alors que je suis ta sœur, que tu as aimée sur terre. Tu ne dois pas aimer une autre que moi, la sœur, la sœur !»

« (3.1) (Paroles prononcées) par Nephthys, elle dit: Souverain parfait, viens vers ta demeure ! Rassérène-toi, tu n'as aucun ennemi. Tes deux sœurs sont à côté de toi en protection de ta couche, t'appelant en pleurant. Retourne-toi sur ta couche pour voir les belles! Parle avec nous, Souverain, notre maître et chasse tout le chagrin qui est dans notre cœur. Tes courtisans divins et humains te contemplent ; présente-leur ton visage, souverain, notre maître, (car) c'est de voir ton visage que vivent nos visages. Ton visage ne saura se détourner de nous. Que ça nous est agréable de te contempler! Je suis Nephthys, ta sœur que tu as aimée. Celui qui s'est rebellé contre toi est tombé, il n'existera plus! Je suis avec toi en protection de ton corps pour toujours et à jamais (...). »

\section{Notice liturgique}

«(5.13) Après la récitation de cela, l'endroit doit être totalement réservé, sans que rien ne puisse être vu ni entendu par quiconque, à l'exception du prêtre-ritualiste et chef, ainsi que le prêtre-setem. Puis, on doit aller chercher deux femmes de belle apparence physique. Les faire asseoir sur le sol, à l'emplacement des vantaux de la cour d'apparition, les noms d'Isis et de Nephthys étant écrits sur leurs épaules. Placer des aiguières de faïence pleines d'eau dans leurs mains droites, et des galettes faites à Memphis dans leurs mains gauches. Faire baisser leur visage. À accomplir à la troisième heure du jour, et de même à la huitième heure du jour. Pas question que tu te relâches dans la récitation de ce manuscrit pendant l'heure de la cérémonie. C'est terminé. ${ }^{63}{ }^{»}$

63. Extrait des «Lamentations d'Isis et de Nephthys » du P. Berlin 2008. Le texte provient sans doute de la région thébaine, et date de l'époque ptolémaïque ; des critères internes permettent de supposer à une rédaction initiale située entre le $\mathrm{VII}^{\mathrm{e}}$ et $\mathrm{IV}^{\mathrm{e}}$ siècles av. J.-C. Voir : R. Faulkner, The Lamentations of Isis and Nephthys, Mélanges Maspero I, 1, 1934, p. 337-348 ; Miriam Lichtheim, Ancient Egyptian Literature, III, p. 116-121 ; pour la traduction, $c f$. Pascal Vernus, Chants d'amour de l'Égypte antique, p. 96-98 (traduction légèrement modifiée ici en ce qui concerne certains passages). Voir en outre Marc Coenen, «New stanzas of the Lamentations of Isis and Nephthys », OLP 31, 2000, p. 5-23. 


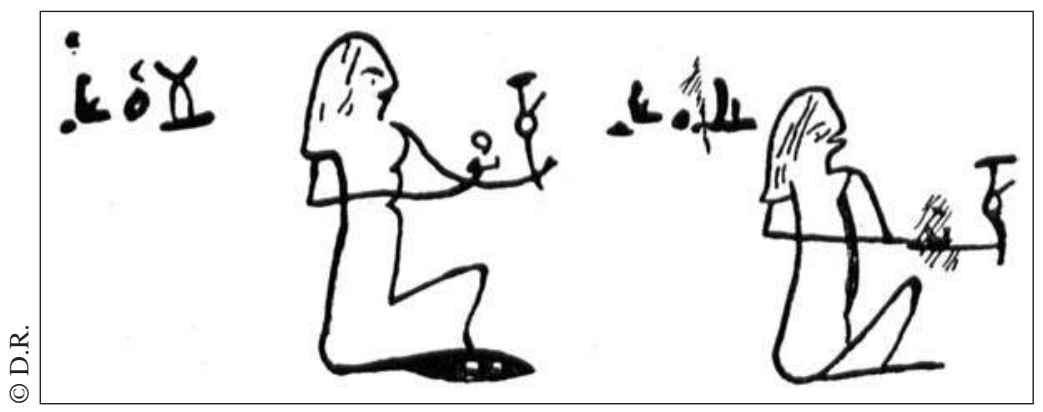

Fig. 2. Les pleureuses, Isis et Nephthys.

Détail d'après Raymond Faulkner, The Lamentations of Isis and Nephthys, Mélanges Maspéro I, 1, Le Caire, 1934.

\section{La cérémonie des « deux oiselles »}

«(1.1) Commencement des stances de la cérémonie des deux oisellesmilans, qui est célébrée dans la demeure d'Osiris-qui-préside-à-l'Occident, le dieu grand, maître d'Abydos, le quatrième mois de la saison de l'inondation, du vingt-deuxième jour au vingt-sixième jour.

On doit réserver la demeure tout entière. On doit aller chercher deux femmes au corps purifié, qui n'ont pas encore enfanté (litt. “qui n'ont pas été ouvertes", c.à.d. qui sont toujours vierges), et qui ont été épilées sur (tout) leur corps, la tête ceinte d'un [bandeau ?], un tambourin dans les mains, et dont les noms ont été inscrits sur les épaules pour Isis et Nephthys ; qu'elles chantent les stances de ce rouleau devant ce dieu. Elles doivent dire : "ô maître Osiris !", quatre fois. »

(...)

«(6.23) Paroles à réciter par les deux femmes-aux-nattes :

Ihy parfait, viens vers ta demeure ! haut, haut, ton dos vers ta demeure, alors que les dieux sont à leur place ${ }^{64}$. Je suis Isis, la femme utile à son frère, ton épouse, sœur de ta mère, viens vite auprès de moi ! Car mon désir est de voir ton visage, puisque je n'ai encore vu ton visage. L'obscurité est ici pour nous, devant mon visage, alors que Rê est dans le ciel. Le ciel se confond avec la terre et crée l'ombre sur la terre aujourd'hui. Mon cœur brûle parce que tu es parti de cette triste manière, mon cœur brûle parce que tu m'as tourné le dos, alors que tu n'avais jamais manifesté cette attitude contre moi. Les deux buttes

64. Vernus, op. cit., p. 107, comprend « de toute ta taille, de toute ta taille, ton dos (tourné) vers ton domaine ! et ce, alors que les dieux seront à leur place »; Assmann, op. cit., p. 220, interprète le passage dans un sens radicalement différent : "Tu t'es éloigné loin de ta maison, bien que les dieux (y) fassent leur devoir ». 
sont bouleversées, les routes sont enchevêtrées, tandis que je suis en quête, afin de te voir. (7.10) Je suis dans une ville sans remparts, je pleure ton amour pour moi, ne reste pas seul! ne reste pas au loin ! Vois, ton fils repousse Tebeha (= Seth) vers le billot (de supplice). ${ }^{65}$ »

Une phase cruciale du drame mythologique est donc jouée par deux actrices, deux jeunes femmes personnifiant Isis et Nephthys (fig. 2). Il se trouve qu'un documentation papyrologique privée (les archives de «Ptolémée le reclus », datant du milieu du $\mathrm{II}^{\mathrm{e}}$ siècle av. J.-C.) nous offre quelques renseignements sur la personnalité de deux de ces «pleureuses » : on y trouve consignées quelques bribes du destin de deux jeunes égyptiennes, des sœurs jumelles, nommées respectivement Taous et Thauès. Ces deux sœurs personnifièrent les déesses lors des funérailles de l'Apis. Elles s'acquittèrent si bien de cette tâche, d'ailleurs, qu'elles furent gardées par le clergé pour accomplir les oblations quotidiennes pour Osiris-Apis, et qu'elles furent appelées à tenir le même rôle de pleureuses, six ans plus tard, pour les funérailles du taureau Mnévis à Héliopolis ${ }^{66}$. L'expression de la tristesse est ici clairement l'affaire de professionnelles.

\section{Pleurs et lamentations lors des funÉrailles FAMiliales : QUELLE PLACE POUR LES ÉMOTIONS INTIMES ?}

La riche documentation provenant des tombes privées éclaire la pratique des chants funèbres et des lamentations dans le cadre des funérailles familiales ${ }^{67}$.

65. Extrait du p. British Museum 10188 / p. Bremner-Rhind. Le texte est daté par le scribe qui en fait copie en «l'an 12 d'Alexandre fils d'Alexandre (Alexandre Aigos) » ( 311 av. J.-C.). Voir Raymond O. Faulkner, The Papyrus Bremner-Rhind, Bibliotheca Aegyptiaca III, 1933, p. 1-32. Trad. : Pascal Vernus, Chants d'amour de l'Égypte antique, Paris, 1992, p. 101-119. Cf. aussi Jan Assmann, Mort et au-delà en Égypte ancienne, Paris, 2003, p. 220-221.

66. Pour ce dossier célèbre, consigné dans les archives des « reclus » du Sérapeum, voir la présentation de Michel Chauveau, L'Égypte au temps de Cléopâtre, Paris, 1997, p. 162-163.

67. L'étude de base demeure celle de Erich Lüddeckens, Untersuchungen über religiösen Gehalt, Sprache und Form der ägyptischen Totenklagen, MDAIK 11, 1943. Voir aussi Jürgen Settgast, Untersuchungen zu altägyptischen Bestattungsdarstellungen, ADAIK 3, Glückstadt, 1963 ; Ursula Rössler-Köhler, s.v. Totenklage, Lexikon der Ägyptologie VI, 1986, col. 657-658. 
L'image et les textes mettent en évidence deux types de manifestation du chagrin. D'une part, des scènes montrent l'affliction personnelle manifestée par des membres de la famille et leurs proches lors des funérailles. D'autre part, on rencontre au sein du cortège funèbre un groupe de femmes exprimant leur peine de façon ostentatoire. Ce groupe des «pleureuses » (qui, en fait, n'est jamais nommé ainsi) accompagne la famille ${ }^{68}$. Parmi les quelques désignations (assez peu explicites) de ces pleureuses ${ }^{69}$, le terme de ouchebout signifie apparemment les « répondantes ${ }^{70}$. Cette appellation pourrait laisser entendre que les lamentations de ce chœur de femmes « répondent » aux plaintes de la famille et de la veuve. Nous noterons que le terme de « pleureuse » (rémjt $)^{71}$ est quant à lui réservé à cette dernière.

Le groupe des pleureuses est très largement illustré dans les scènes figurées (le thème se développe notamment dans les tombes thébaines à partir de la XVIII ${ }^{\mathrm{e}}$ dynastie) ${ }^{72}$, et l'on peut, comme l'a fait autrefois Marcelle Werbrouck ${ }^{73}$, observer toute une palette d'attitudes : bras levés au ciel, larmes ${ }^{74}$, poitrine dénudée ${ }^{75}$, prostrations. Ces attitudes

68. Voir Marcelle Werbrouck, Les pleureuses dans l'Égypte ancienne, Fondation égyptologique Reine Elisabeth, Bruxelles, 1938, ancienne (et plaisante) étude, toujours utile, faisant une large part à l'iconographie. $C f$. en outre Christine Seeber, sv. Klagefrau, Lexikon der Ägyptologie III, 1980, col. 443-447.

69. Les désignations d'Isis et Nephthys en tant que plaignantes sont quant à elles assez variées, le terme de $\operatorname{djeret}(y)$ étant le plus commun. $C f$. $W b \mathrm{VI}$, sv. «Klageweib» et «Klagefrau ». L'appellatif de « deuillante(s)» (jakebty et var.) est aussi communément employé, $c f$. Chr. Leitz (éd.), LÄGG I, OLA 110, 2002, p. 115. On utilise aussi le terme de «plaignante» (hayt), cf. Leitz, $L \ddot{A} G G$ V, OLA 114, 2002, p. 8-9, tiré du substantif ha « la plainte ».

70. Wb I 373.1 (XIX ${ }^{\mathrm{e}}$ dynastie) ; Erich Lüddeckens, Totenklagen, MDAIAK 11, 1943, p. 111-112. Marcelle Werbrouck, Les pleureuses dans l'Égypte ancienne, p. 11-12.

71. $W b$ II 417.17.

72. Le cortège des pleureuses figure également dans les vignettes du Livre des Morts, par exemple: The Book of the Dead. Facsimile of the Papyrus of Ani in The Bristish Museum, London, 1890, pl. 6.

73. Op. cit., p. 143-159.

74. Le premier cas de larmes dessinées sur les joues des pleureuses relevé par Marcelle Werbrouck, Les pleureuses dans l'Égypte ancienne, p. 138, provient de la tombe de Houy (TT n ${ }^{\circ} 54$; règne de Touthmès IV / Amenhotep III).

75. Sur le dévoilement des seins dans le cadre funéraire romain, voir la contribution de Francesca Prescendi dans le présent volume. En Égypte, nous 
concernent plus particulièrement ce groupe de femmes, mais pas exclusivement toutefois ${ }^{76}$. La gestion de la mort, qui implique la déploration et les larmes ${ }^{77}$, est universellement une tâche tout spécialement féminine ${ }^{78}$. Dans le monde méditerranéen, les exemples de cortèges de pleureuses sont multiples, de l'antiquité jusqu'à nos jours. Ernesto De Martino s'y est intéressé dans des études fameuses ${ }^{79}$, qui tissent un lien entre les pratiques antiques et certains comportements modernes. En Égypte actuelle, à l'instar d'autres pays musulmans ${ }^{80}$, le cortège des femmes en deuil est encore un spectacle saisissant pour l'observateur occidental (fig. 3) ${ }^{81}$. Nous

avons déjà constaté dans les Textes des Pyramides (voir ci-dessus) l'association de ce geste à la déploration d'Osiris. Hérodote (II, LXXXV, extrait cité ci-après) mentionne aussi le dénudement de la poitrine. Cette attitude rituelle est largement confirmée par l'iconographie. Cf. tout spécialement Marcelle Werbrouck, Les pleureuses dans l'Égypte ancienne, fig. 56 p. 101 : dans un cortège de pleureuses figurées sur un cercueil de la XXI ${ }^{\mathrm{e}}$ dynastie (Bruxelles E 5881), un groupe de femmes présente leurs seins, qu'elles saisissent dans leurs mains, tandis que l'une d'entre elles, aux cheveux dénoués, la poitrine dénudée vue de face, lève spectaculairement les bras au ciel. Nous noterons encore que le dévoilement des seins dans le cadre cultuel est connu par ailleurs, cf. par exemple Serge Sauneron, Les fêtes religieuses d'Esna aux derniers siècles du paganisme, IFAO, Le Caire, 1962, p. 42 (fête du 29 Athyr «présentation des offrandes » en l'honneur de la déesse Nebtou).

76. Le groupe des femmes en pleurs, que l'on rencontre aussi bien dans la procession (sur terre ou sur barque) que devant le catafalque ou la momie dressée devant la tombe, semble exclure le plus souvent une présence masculine. Toutefois, des groupes d'hommes en lamentation sont aussi attestés, représentés parfois à côté du groupe des femmes, parfois se mêlant à celui-ci.

77. Gary L. Ebersole, «The Functions of Ritual Weeping Revisited : Affective Expression and Moral Discourses », in John Corrigan (éd.), Religion and Emotion, Oxford, 2004, p. 185-222.

78. Voir sur ce sujet les références données par Anne-Caroline Rendu dans le présent volume (note $\mathrm{n}^{\circ} 7$ de sa contribution).

79. Notamment Morte et pianto rituale nel mondo antico, Torino, 1958.

80. Voir spécialement Marie Virolles-Souibès, «Les gestes du deuil. Exemples algériens », Gestes et images 8-9, 1991, p. 117-142.

81. C'est presque un lieu commun que d'insister sur ce point: cf. par exemple les remarques de Marcelle Werbrouck, Les pleureuses dans l'Égypte ancienne, p. 123 « (...) les pleureuses peuvent donner libre cours à cette désolation de commande qui semble avoir été transmise inchangée des âges reculés de l'époque pharaonique aux fellahs de nos jours ». 


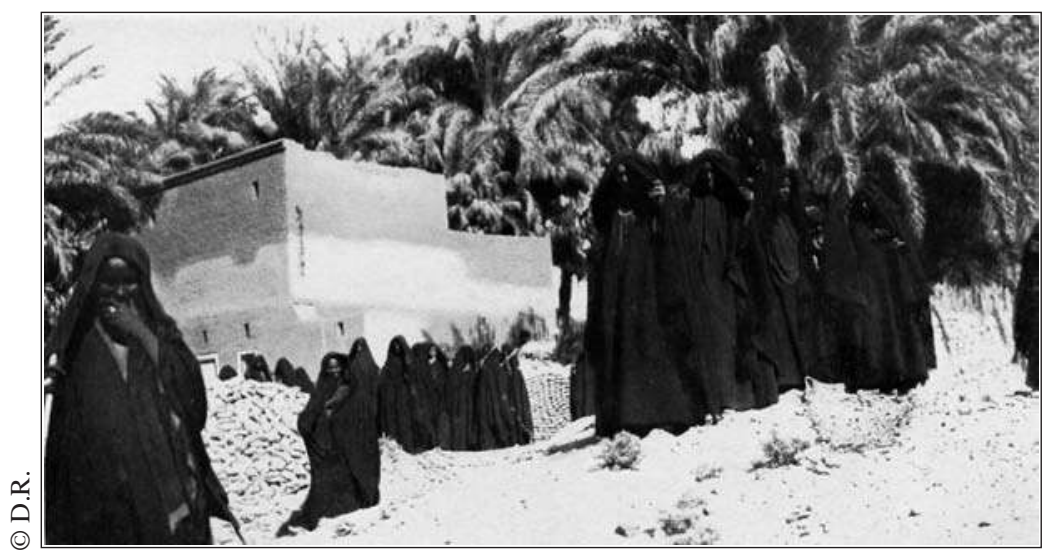

Fig. 3. Le cortège des pleureuses de Karnak.

D'après George Legrain, Louqsor sans les pharaons, Paris, 1914, p. 213-214.

relèverons, à ce propos, les observations d'un ethnographe amateur, faites en Haute Égypte (vers 1930) :

«(...) La fonction des pleureuses est aussi d'antique tradition. Un fellah est mort. Aussitôt les cris des femmes l'annoncent à tous les environs. On va chercher la civière commune qui se trouve près du gourn hors du village. À l'intérieur de la maison, des femmes lavent le corps à l'eau chaude, tandis qu'à l'extérieur s'élèvent les complaintes des pleureuses. En leur oraison funèbre, elles vantent la beauté et les qualités du défunt, elles reprochent à Dieu de l'avoir rappelé ; elles interpellent le défunt, sa femme, ses enfants, la mort elle-même... Ces complaintes semblent improvisées ; elles ne le sont pas plus que les larmes de ces professionnelles, enveloppées de grandes mélayas destinées à cette cérémonie, le visage noirci ou bleui en signe de deuil, la tête couverte de boue... Elles mènent la macabre sarabande, se lèvent, battent des mains, se balancent et retombent épuisées, ralentissant, puis reprenant leurs cris, parmi les femmes. Ici, les femmes priment (...). ${ }^{82}{ }^{»}$

Conforme à l'ensemble des témoignages ethnographiques, anciens ou modernes, cet exemple nous met en présence d'une représentation théâtralisée ; une dramaturgie codifiée, exploitant un catalogue

82. Henri Habib Ayrout S. J., Mours et coutumes des Fellahs, Payot, Paris, 1938, p. 123. De telles cérémonies sont encore communes de nos jours, surtout dans la campagne égyptienne. 
d'attitudes. Les pleurs deviennent alors ce que Marcel Mauss qualifiait d' « expression obligatoire des sentiments $»{ }^{83}$.

En conséquence, cette prise en charge du chagrin par des groupes spécifiques tend à ne pas laisser grande place - du moins dans la documentation - aux expressions personnelles. Elle tendrait d'ailleurs - si l'on suit l'analyse d'Ernesto de Martino - à canaliser les douleurs des personnes frappées par le deuil. Néanmoins, malgré les pleureuses «mandatées », les émotions individuelles ne sont pas annulées; elles se manifestent en adoptant des codes précis ${ }^{84}$. Hérodote nous a laissé quelques observations sur les lamentations (thrènoi) privées égyptiennes :

«Voici leurs deuils (thrènoi) et leurs cérémonies funèbres (taphai): dans la famille qui perd un homme de quelque considération, toutes les femmes de la maison se couvrent de boue la tête et même le visage ; puis elles laissent le cadavre dans la maison et courent par la ville en se frappant la poitrine, le sein nu, et la robe retroussée, retenue par une ceinture ; toutes leurs parentes se joignent à elle. Les hommes se frappent

83. Marcel Mauss, «L'expression obligatoire des sentiments, Journal de psychologie 18, 1921, texte repris dans Essais de sociologie, Paris, 1971, p. 81-88. Même constat, en ce qui concerne l'expression de la douleur du deuil par Philippe Ariès, L'homme devant la mort, Paris, 1977, p. 320, et notamment en rapport au « deuil à pleureuses » qui subsistait en France au XVIII ${ }^{\mathrm{e}}$, et dans l'Europe méditerranéenne (de la Sicile et la Calabre jusqu'à la Grèce et au monde balkanique) jusqu'à l'époque moderne. Cf. aussi Marc Leproux, Du berceau à la tombe. Contributions au folklore charentais, PUF, Paris, 1959, qui signale (p. 271) des pleureuses à la fin du XIX siècle encore dans les villages de Combiers et Roumazières. Pour Ariès (loc. cit.), « au fil du temps (ces traditions) apparaissent de plus en plus comme des pratiques rituelles d'où la spontanéité était tout à fait absente ». Voir aussi Arnold van Gennep, Manuel de folklore français contemporain, I/2. Du berceau à la tombe. Mariagefunérailles, Picard, Paris, 1938 (rééd. 1980), p. 668-669 et p. 681-686. Van Gennep relève la disparition progressive, surtout depuis la guerre de 19141918 , de ces « cris et hurlements » poussés par les femmes, des cris parfois «effroyables » (p. 681). Les hurlements de douleur sont aussi accompagnés d'une récitation (le lamento), prononcée par le chœur des femmes.

84. Sur les manifestations de chagrin dans le cadre de «l'expression sociale des émotions » et leurs implications, voir David Le Breton, Les Passions ordinaires. Anthropologie des émotions, Payot, Paris, 2004, p. 147-158 (1 $1^{\text {re }}$ éd. Armand Colin, Paris, 1998). 
et se lamentent de leur côté, dans une tenue semblable. Cela fait, on emporte le corps pour le faire embaumer. ${ }^{85}$ »

Cette manifestation d'affliction au cours de laquelle on se salit, peut être directement rapprochée des indications offertes par un long texte funéraire tardif, qui évoque lors des funérailles « les hommes, leurs membres couverts de poussière, et les femmes ointes de boue (...) leurs yeux enfiévrés et mouillés (tandis que) leurs voix sont chaudes à lancer des plaintes $(. ..){ }^{86}$. Un tel rite d'autoagression, au cours duquel la violence ressentie face à la mort se traduit par des gestes brutaux que s'inflige à lui-même le «deuillant », est là encore un phénomène assez largement constaté ; par exemple, comme le rappelle Walter Burkert, « la lamentation funèbre - pleurer et hurler, se déchirer les vêtements, s'arracher les cheveux, se griffer le visage et se frapper la poitrine - ; puis se "souiller" soi-même, maiesthai, se barbouiller le visage, s'asperger la tête avec de l'argile, de la terre ou des cendres (...) » sont des façons de faire bien attestées dans la culture grecque ancienne ${ }^{87}$. Un parcours dans la littérature antique $^{88}$, dans les textes bibliques ${ }^{89}$, tout comme dans l'ethnographie nous amènerait, du reste, à collectionner des manifestations de tristesse analogues ${ }^{90}$. En Égypte, ces manifestations paroxystiques

85. Hérodote, II, LXXXV, trad. A. Barguet. On rapprochera encore ce passage de celui consacré aux rites de déplorations d'Osiris (qu'Hérodote - à son habitude - évite de nommer) observés à Busiris : après le sacrifice (thusia), tous les participants se frappent; Hérodote précise que « les Cariens habitant en Égypte font encore d'avantage, puisqu'ils vont jusqu'à se taillader le front à coups d'épées ; par là, ils se font connaître comme étant étrangers (xenoi) et non pas Égyptiens » (II, 61).

86. François René Herbin, Le livre de parcourir l'éternité (OLA 58), Leuven, 1994, p. 62 (V.22-24) et commentaire p. 210-211.

87. Walter Burkert, Homo necans. Rites sacrificiels et mythes de la Grèce ancienne, Les Belles Lettres, Paris, 2005, p. 57-58.

88. Sur les attitudes féminines violentes lors du deuil à Rome, voir la contribution de Francesca Prescendi dans le présent volume.

89. Exempli gratia: 2 Samuel 15.30 (pleurs, tête voilée, pieds nus, tunique déchirée, tête couverte de terre) ; 2 Samuel 19.5 (visage voilé) ; Lévitique 10.5 (cheveux défaits, vêtements déchirés).

90. En son temps, Emile Durkheim s'était déjà arrêté sur la question des violences auto-infligées dans les rites de deuil, cf. Les formes élémentaires de la vie religieuse, Paris, 1912, chapitre V « les rites piaculaires et l'ambiguïté 
du chagrin côtoient d'autres attitudes corporelles, tout autant emblématiques de la souffrance ${ }^{91}$. On signalera à cet égard que la posture consistant à lever les deux bras au ciel est d'usage tant pour exprimer le chagrin que la joie ${ }^{92}$ : cette position signale un état émotionnel intense, quelle que soit sa connotation. Enfin, on notera tout particulièrement le rôle de la chevelure : les cheveux défaits des femmes signalent une situation de détresse émotionnelle ${ }^{93}$. Il semble en outre que des signes de deuil puissent être exprimés avec plus de retenue par l'absence de soins de toilette; on remarquera ainsi certaines figurations (surtout sur ostraca) montrant un visage royal mal rasé, signe que l'on peut considérer sans doute comme une marque visible de deuil ${ }^{94}$.

de la notion du sacré » : « hommes et femmes, saisis par une véritable frénésie, couraient, s'agitaient, se faisaient des blessures, avec des bâtons pointus ; les femmes se frappaient les unes les autres sans qu'aucune ne cherchât à se garantir des coups » (p. 559, in édition PUF, Paris, 1968). Citons encore la description saisissante faite en Corse par Lorenzi de Bradi, transmise par Arnold van Gennep, Manuel de folklore français contemporain, I/2.1938, p. 668-669 : «Soudain des cris s'élèvent. Le malade vient d'expirer. On se presse. Autrefois on renversait les chaises et les tables. Les femmes sont transformées en furies. Elles crient sauvagement, dénouant leurs cheveux, s'égratignant le visage (...) peu à peu, l'apaisement se fait. Alors, l'une des femmes se penche sur le cadavre et, se balançant lentement, elle chante d'une voix traînante et criarde une sorte de liturgie, qui dit les qualités du mort. Le rythme est saccadé, rude. Aucune larme d'émotion. Le visage est sec (...). »

91. Brigitte Dominicus, Gesten und Gebärden in Darstellungen des Alten und Mittleren Reiches, SAGA 10, 1994, p. 65-72.

92. Dominicus, op. cit., p. 58-61. Le même signe hiéroglyphique de l'homme aux deux bras levés (Sign List Gardiner A 28) sert d'ailleurs de déterminatif tant pour des verbes exprimant la joie que le chagrin ; voir Philippe Collombert, Le mastaba de Mérérouka. Paléographie hiéroglyphique, à paraître prochainement à l'IFAO.

93. Par exemple : Nina de Garis Davis et Alan H. Gardiner, The Tomb of Amenemhet ( $\left.n^{\circ} 82\right)$, London, 1915, pl. XXIV. On peut aussi relever que le signe hiéroglyphique de la mèche de cheveux (Gardiner Sign List D 3) est utilisé notamment comme déterminatif pour des termes liés au deuil ou aux plaintes.

94. Par exemple : William Peck, Dessins égyptiens, Hermann, Paris, 1980, fig. 31 ; Jacqueline Vandier d'Abbadie, Catalogue des ostraca figurés de Deir el-Médineh, DFIFAO II/2, Le Caire, 1937, n 2568 et 2569, pl. LXXII/LXXIII, p. 116-117. W. Westendorf, s.v. Trauer, Lexikon der Ägyptologie VI, 1986, col. 744-745. 


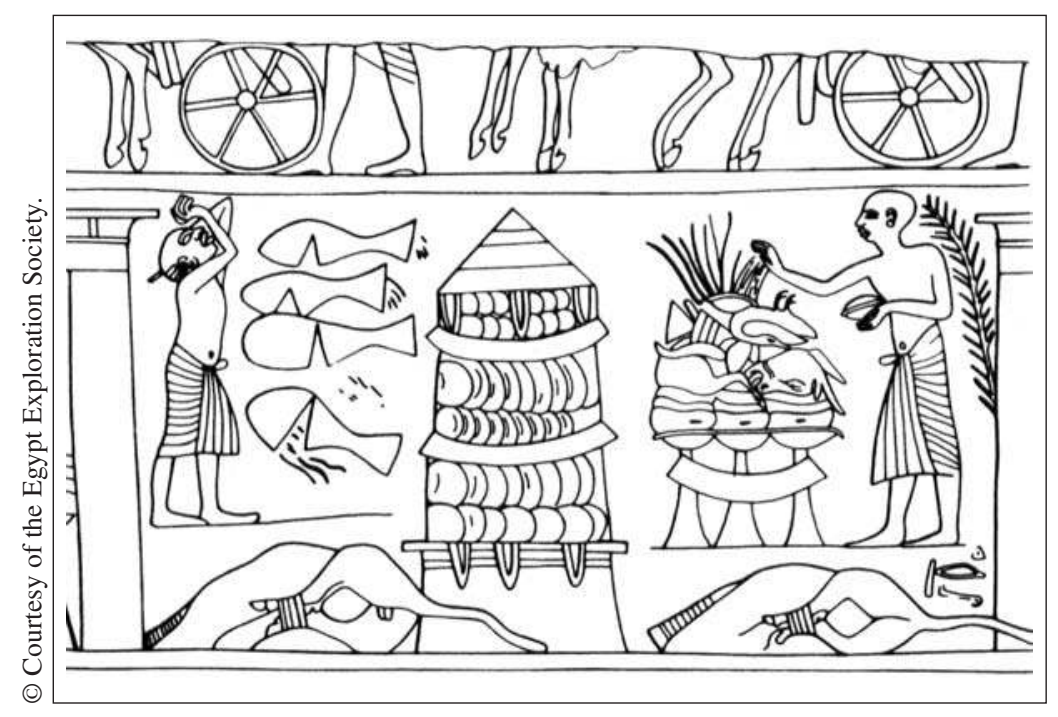

Fig. 4. Bris de vases lors des rites funèbres.

Détail d'après Geoffrey T. Martin, The Memphite Tomb of Horemheb, EES, London, 1989, pl. 123.

La violence gestuelle engendrée par l'émotion du deuil n'est pas seulement adressée à l'humain : elle peut aussi s'exprimer par un bris d'objets. On connaît une très ancienne procédure magique consistant à briser une vaisselle rouge ( « briser les pots rouges » : sédj déshérout). Ce rite prend place dans des procédures d'exécration de l'ennemi, et manifestement aussi lors des funérailles, à la fin du rituel des offrandes ${ }^{95}$. Or l'iconographie nous fait connaître des scènes montrant un prêtre, lors de cérémonies funèbres, qui se lamente devant un tas de pots préalablement brisés (fig. 4$)^{96}$. Ce moment du rite funéraire n'est pas facile à interpréter, car ces scènes ne sont pas soutenues par des légendes explicites. On a généralement interprété le bris

95. Jacobus van Dijk, «Zerbrechen der roten Töpfe », Lexikon der Ägyptologie VI, 1986, col. 1389-1396. Cf. Jan Assmann, Martin Bommas et Andres Kucharek, Altägyptische Totenliturgien, 2, Totenliturgien und Totensprüche in Grabinschriften des Neuen Reiches, Heidelberg, 2005, p. 116-117.

96. Par exemple : Geoffrey T.Martin, The Memphite Tomb of Horemheb, Commander-in-Chief of Tut'Ank Amun, EES, London, 1989, pl. 123. 
funéraire des vases comme un rite apotropaïque ${ }^{97}$ : le vase rouge symboliserait l'ennemi séthien à éliminer. Cette explication ne permet pas toutefois de comprendre les gestes d'affliction accomplis devant ces vases, desquels s'échappe un flot de liquide. Il n'est pas impossible que, dans le cadre funéraire, le bris des cruches prenne une coloration symbolique différente de celle des rites magiques d'exécration. Mutatis mutandis, la pratique du bris des jarres dans le cadre du deuil est connue encore dans l'Égypte moderne, comme on le constate dans ce chant funèbre pour une défunte qu'avait relevé Georges Legrain, au début du $\mathrm{XX}^{\mathrm{e}}$ siècle :

«Pourquoi te retournes-tu sur ta couche ? Les matelas et les draps de soie ne te suffisent-ils pas ? Soulève-toi pour rajuster ta coiffe, et promène-toi dans ta maison pour que je te contemple (...) (chant accompagnant le bris des vases-zîrs) : Quelle tristesse ! nous allons retourner les vases. Seule, ta maison sera ruinée au milieu des nôtres (...).$^{98}$ »

Si nous nous tournons vers les images du deuil, nous voyons que les scènes figurées dans les tombeaux des particuliers ménagent dès l'Ancien Empire une place aux représentations du cortège funèbre. On y remarque parfois la présence de membres éplorés de la famille et de la maisonnée ; ainsi dans le document suivant, tiré du monument funéraire d'Idou :

«Ô (mon) père (bien) aimé !

Ô mon maître, prends-moi vers toi !

Ô mon maître (bien) aimé !

Procession (litt. sortie) de ses gens (serfs) en larmes. »

(Texte du montant droit, légendant la figuration des « deuillants »99)

Ces légendes accompagnent respectivement quatre registres, qui montrent chacun un groupe de personnages (fig. 5). Plusieurs attitudes emblématiques de la douleur sont représentées : un homme se frappe la tête avec un bâton, un autre semble s'arracher les cheveux ; plusieurs personnages sont au sol, et l'on tente de les soutenir ; comme

97. Cf. van Dijk, op. cit., col. 1393.

98. Georges Legrain, Louqsor sans les pharaons, Paris, 1914, p. 213-214 (cf. aussi spécialement p. 213-222 « Chansons funèbres »).

99. Texte provenant de Guizeh, tiré du mastaba d'Idou (VIe dynastie). Voir William K. Simpson, The Mastabas of Qar and Idu, Giza Mastabas vol. 2, Museum of Fine Arts, Boston, 1976, p. 22 et fig. 35 ; pls. XVIII-XIX. 


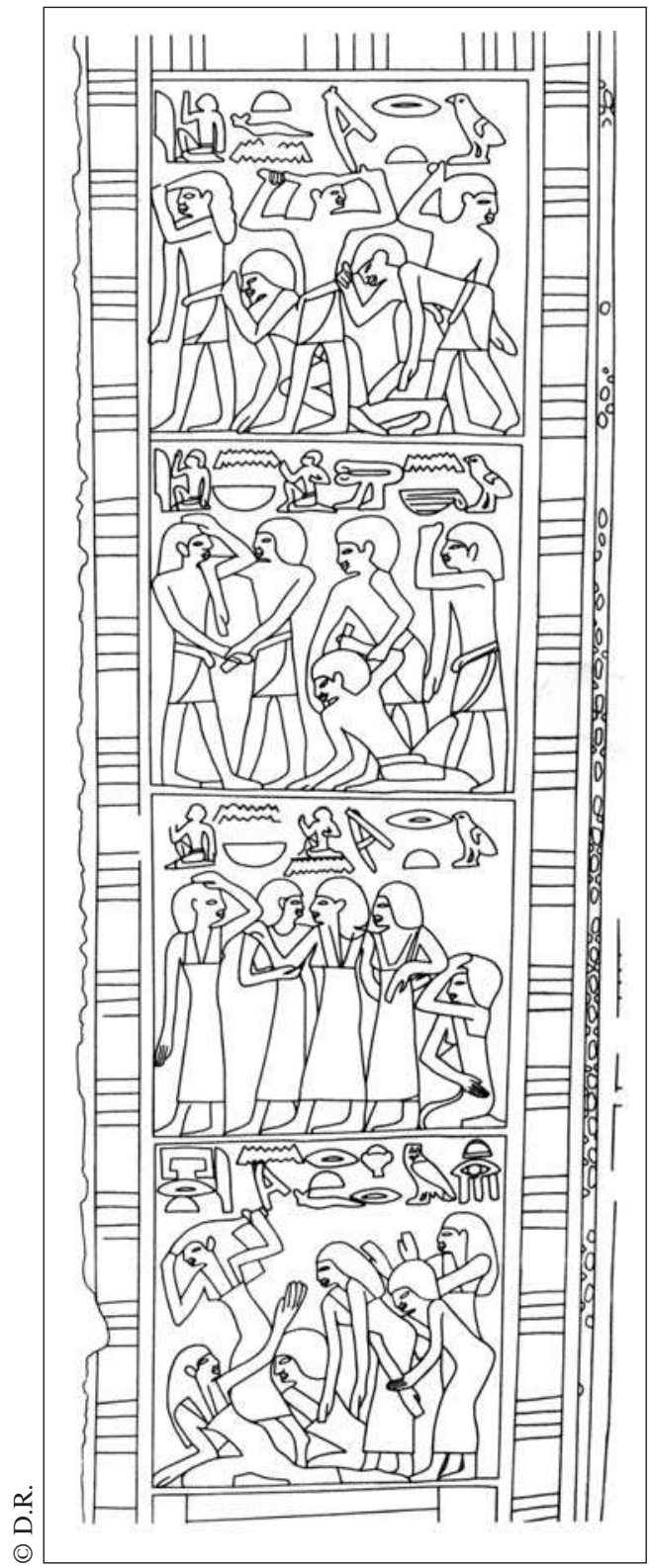

Fig. 5. Scènes de funérailles dans le mastaba d'Idou. D'après William K. Simpson, The Mastabas of Qar and Idu, Giza Mastabas, vol. 2, Boston, 1976, pl. XVIII-XIX. 
l'indique la légende du registre inférieur, c'est là toute la maisonnée du notable qui est en proie au chagrin. Cette expression collective (et codifiée) du chagrin s'ajoute à la présence de pleureuses rituelles, lesquelles sont représentées sur un autre panneau du tombeau et désignées par le terme djéret ${ }^{100}$. Ce type de représentation est assez commun dans les scènes de l'Ancien Empire; les discours mis en légendes n'insistent pas sur la douleur portée par une plainte verbale ${ }^{101}$.

Ultérieurement, les scènes funéraires des tombes privées du Nouvel Empire (et tout particulièrement celles de la nécropole thébaine) offrent une documentation considérable sur les scènes d'affliction collective lors du cortège funèbre. On y rencontre non seulement les pleureuses, mais encore différents groupes adoptant une large palette d'attitudes, allant du chagrin ostentatoire au recueillement douloureux. Les scènes funéraires du Nouvel Empire ménagent aussi une place aux lamentations de la veuve. Tout se passe comme si la période amarnienne, qui connaît une inflexion nouvelle également en ce qui concerne les rites funéraires, avait brisé un interdit entourant la commémoration de la déploration tragique ${ }^{102}$. Les accents de ces chants plaintifs sont en tout point comparables aux chants liturgiques de déploration d'Osiris, chants qui en sont les prototypes. On remarquera aussi que les déplorations ne ménagent aucune place à la consolation; au contraire, elles insistent sur la cruauté de la perte $^{103}$. Les lamentations des pleureuses font écho aux plaintes de

100. M. Werbrouck, Les pleureuses, op. cit., p. 15-16.

101. J. Assmann (dans Mort et au-delà, op. cit., p. 214-215), suppose que la douleur et le chagrin, en tant qu'aspects négatifs de la mort, n'avaient pas encore place dans l'économie funéraire de l'Ancien Empire, période durant laquelle la forte croyance en l'immortalité interdisait de se focaliser sur ces aspects.

102. Pour cette idée, cf. également Jan Assmann, Mort et au-delà en Égypte ancienne, p. 215.

103. Remarquons que c'est aussi le cas dans les épigrammes funéraires grecques qui, en Égypte en tout cas, se nourrissent entre autres de traditions et de façons de faire locales. À ce sujet, cf. le recueil publié par Etienne Bernand, Inscriptions métriques de l'Égypte gréco-romaine, Paris, 1969 ; cf. p. 33-41 pour les «sentiments » témoignés dans ces inscriptions funèbres. 
la veuve, comme dans cet extrait tiré de la tombe de Néferhotep à Thèbes ${ }^{104}$ (fig. 6) :

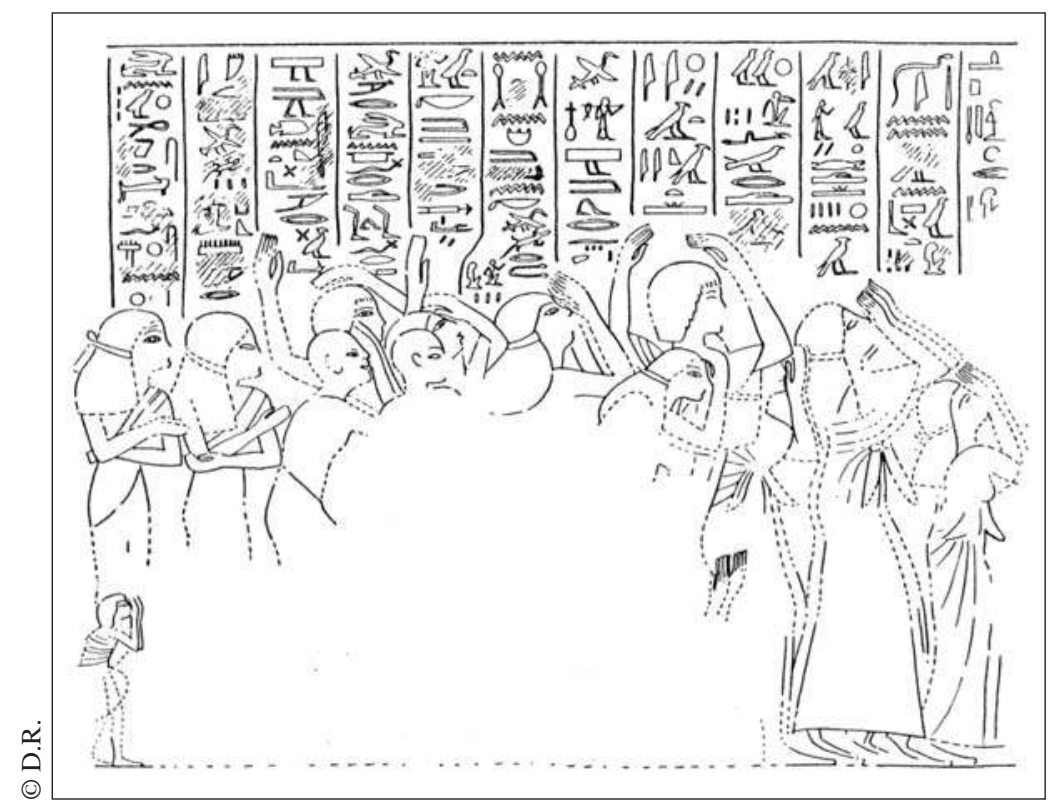

Fig. 6. Lamentations aux funérailles de Néferhotep.

D'après Erich Lüddeckens, Totenklagen, MDAIAK 11, 1943, p. 109-110.

« Je suis ta sœur, Méryt-Rê ! Ô grand, n’abandonne pas Méryt-Rê ! Ton état est bon ! Toi, bon père, tu es mien. T'en aller, comment peux-tu faire cela ? Je vais seule, vois, je suis derrière toi ! Toi qui aimais bavarder avec moi, tu te tais, tu ne parles plus !»

« Paroles à dire par les "pleureuses" (litt. ici ouchébou "les répondantes") : Douleur !, Douleur (imaou) ! Sain, sain, sain, sain ! Plainte sans cesse ! Ah ! Perte (ta aqyt) ! Le bon berger est parti au pays de l'éternité ; lui dont les gens étaient nombreux, il est dans le pays qui aime la solitude. Lui qui aimait à bouger (litt. "ouvrir") ses pieds pour marcher, il est

104. Texte tiré de la tombe thébaine ${ }^{\circ} 49$ datant du règne d'Ay (XVIII ${ }^{\mathrm{e}}$ dynastie), vers 1320 av. J.-C.

Voir Jan Assmann, Mort et au-delà en Égypte ancienne, p. 215-216 ; Erich Lüddeckens, Totenklagen, MDAIAK 11, 1943, p. 109-110 8 (Nr. 48), 111-113 (Nr. 49) et pl. 24. 
entravé, emmailloté, bloqué ! Lui qui aimait se vêtir de belles étoffes, il dort dans le vêtement d'hier!»

L'insistance dramatique sur la cruauté de la mort est aussi particulièrement poignante dans la tombe de Nefersékherou à Zawiyet Sultan ${ }^{105}$ (fig. 7) :

«Moutnéferet (= la veuve) dit : Celui qui était de bon conseil, le silence s'est abattu sur lui. Celui qui veillait est dans le sommeil. Celui qui ne dormait pas la nuit est inerte le jour!

Les "pleureuses" (tchésout) disent: la maison de ceux qui sont à l'Occident est profonde et sombre. Elle n'a ni porte ni fenêtre, pas de lumière pour l'éclairer, pas de vent du nord pour rasséréner le cœur. Là, le soleil ne se lève pas. Ils sont endormis tout le temps, car l'obscurité s'étend au jour. Hélas $(y h)$, comme le bon (= le défunt) serait bien portant si on pouvait respirer l'air !

Moutnéfert (= la veuve) dit : Celui qui avait une voix forte se tait, il ne peut plus parler. Celui qui était maître de lui (litt. "celui qui comptait son corps") est inconscient (litt. "ne sait plus"). Hélas! Combien est misérable celui qui dort, couché pour l'éternité. Hélas ! si la couche qui te porte pouvait faire ce qu'a fait ta nourrice, qui te retournait et te tirait de ton sommeil, pour que tu t'éveilles et entendes ma voix ! Le berger a été emmené dans le pays de l'éternité-neheh, dans la place de l'infinité-djet. Dissimulés (litt. shétayt "mystérieux") sont ceux de l'Occident, et pénible ( $q s n)$ est leur condition. Il est immobile, celui qui est allé à eux. Il ne peut pas dire sa condition. Il repose dans sa place solitaire, et l'éternité est à lui dans les ténèbres (...). »

Cette parole de douleur, prononcée par la veuve de Nefersékhérou, ne semble vouloir s'accommoder d'aucune consolation. Elle apparaît dans un monument qui a également livré un représentant célèbre d'un genre littéraire dont le propos est précisément de s'exprimer sur la brièveté de l'existence, tout en ne laissant place à aucun espoir précis quant à l'au-delà : les « chants des harpistes » 106 , déclarations épicuriennes avant la lettre, étaient vraisemblablement psalmodiés durant les banquets funéraires; ils décrivent un au-delà sombre,

105. Texte tiré du tombeau de Nefersékherou à Zawiyet Sultan en Haut Égypte, datant du règne de Ramsès II, (XIX ${ }^{\mathrm{e}}$ dynastie). Voir Jürgen Osing, Das Grab des Nefersecheru in Zawiyet Sultan, ÄV 88, Mainz, 1992, p. 54-55 et pl. 36, cf. Jan Assmann, Mort et au-delà en Égypte ancienne, p. 216.

106. Miriam Lichtheim, "The Song of the Harpers », JNES 4, 1945, p. 178-212; Jan Assmann, L̈̈ II, 1977, col. 972-982; en dernier lieu: Jürgen Osing, Aspects de la culture pharaonique, Paris, 1992, chap. I « Les chants du harpiste au Nouvel Empire ». 


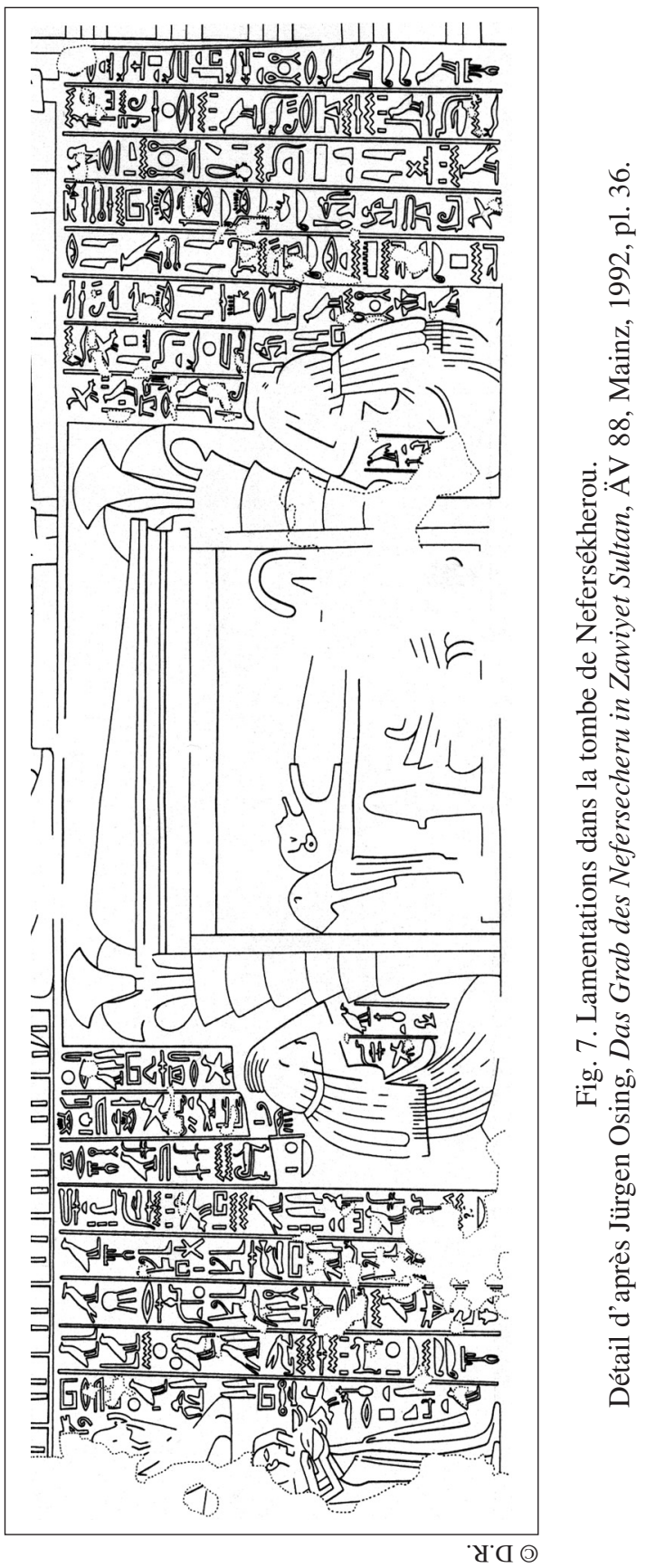


duquel l'on ne revient pas, et, inversement, exhortent l'homme à faire la fête et à profiter de la vie ${ }^{107}$. Dans ces chants, le regard désenchanté jeté sur le trépas introduit comme corollaire direct la célébration exacerbée de l'existence, un motif qui s'accorde bien avec la théologie naturaliste du Nouvel Empire (exaltation du don de vie prodigué par le dieu céleste).

\section{REMARQUES CONCLUSIVES}

L'examen de la documentation égyptienne auquel on vient de se livrer ne lève apparemment pas le voile sur les émotions intimes qui auraient pu être ressenties par les particuliers lors des funérailles. Tout semble réduit à des modèles collectifs. Mais peut-il en être autrement? L'occasion de la mort est, comme on le sait, le moment privilégié pour les expressions émotionnelles collectives. Comme l'écrivait autrefois Maurice Halbwachs : «Quand l'émotion s'exprime, cette expression est matérielle, et le groupe a prise directement sur elle ». Les manifestations émotionnelles égyptiennes s'inscrivent dans ces manifestations funéraires «strictement réglées par la coutume » ${ }^{108}$. Dans la perspective sociologique de l'école de Chicago, Irving Goffman était de son côté amené à traiter d'une vie sociale qui, tout entière, serait « un théâtre » ${ }^{109}$. En forçant le trait, on arriverait vite à penser que la vie émotive, que l'on pense intime,

107. Un autre cas de «pessimisme » témoigné envers le monde funéraire est connu par un texte littéraire de la XII ${ }^{\mathrm{e}}$ dynastie, qui met en scène un homme et son «âme »-ba, en proie à une discussion angoissée. Voir Odette Renaud, Le dialogue du Désespéré avec son âme, CSEG 1, Genève, 1991. Bernard Mathieu, «Le dialogue d'un homme avec son âme », Égypte Afrique \& Orient 19, 2000, p. 17-36. Les propos du ba sont imprégnés de la pensée hédoniste que l'on peut constater de façon comparable dans les chants du harpiste.

108. Maurice Halbwachs, «L'expression des émotions et la société », in Échanges sociologiques, Paris, 1947, repris dans Classes sociales et morphologies, Paris, 1972, p. 164-173.

109. Erving Goffman, La mise en scène de la vie quotidienne. 1. La présentation de soi, Les éditions de minuit, Paris, 1973 et La mise en scène de la vie quotidienne. 2. Les relations en public, Les éditions de minuit, Paris, 1973. 
n'est remplie que d'émotions de «façade », de sentiments convenus, appris, copiés.

La documentation égyptienne nous met en présence d'un ensemble d'émotions «exécutées » en public: manifestations silencieuses (tristesse, abattement) ou au contraire démonstrations bruyantes (pleurs, cris) ; ces manifestations sont d'évidence organisées (cortège des pleureuses). Il serait aléatoire, voire de mauvaise méthode, de vouloir déterminer les parts respectives de la tristesse individuelle (que l'on supposerait « réellement éprouvée ») et collective (qui ne serait que «rituellement exécutée », c'est-à-dire jouée). Rien ne prouve en effet que les émotions collectives et obligatoires sont moins intimement éprouvées que celles que l'on peut ressentir spontanément, hors de toute situation codifiée au préalable. Comme on l'a $\mathrm{vu}$, le modèle égyptien de la situation de détresse émotionnelle éprouvée par le survivant face au décès de l'être aimé est fourni par le mythe d'Osiris. Ce prototype de l'expérience osirienne offre à la société comme à l'individu un référent pour l'expérience de la souffrance et son expression. Il propose en plus les solutions rituelles qui permettent d'affronter et de vivre le deuil.

Université de Genève

Faculté des Lettres

Département des Sciences de l'Antiquité

2 , rue de Candolle

CH-1211 Genève 4 